\title{
Berry skin development in Norton grape: Distinct patterns of transcriptional regulation and flavonoid biosynthesis
}

Mohammad B Ali ${ }^{1,4}$, Susanne Howard ${ }^{1}$, Shangwu Chen ${ }^{3}$, Yechun Wang ${ }^{2}$, Oliver Yu², Laszlo G Kovacs', Wenping Qiu ${ }^{1 *}$

\begin{abstract}
Background: The complex and dynamic changes during grape berry development have been studied in Vitis vinifera, but little is known about these processes in other Vitis species. The grape variety 'Norton', with a major portion of its genome derived from Vitis aestivalis, maintains high levels of malic acid and phenolic acids in the ripening berries in comparison with V. vinifera varieties such as Cabernet Sauvignon. Furthermore, Norton berries develop a remarkably high level of resistance to most fungal pathogens while Cabernet Sauvignon berries remain susceptible to those pathogens. The distinct characteristics of Norton and Cabernet Sauvignon merit a comprehensive analysis of transcriptional regulation and metabolite pathways.

Results: A microarray study was conducted on transcriptome changes of Norton berry skin during the period of 37 to 127 days after bloom, which represents berry developmental phases from herbaceous growth to full ripeness. Samples of six berry developmental stages were collected. Analysis of the microarray data revealed that a total of 3,352 probe sets exhibited significant differences at transcript levels, with two-fold changes between at least two developmental stages. Expression profiles of defense-related genes showed a dynamic modulation of nucleotide-binding site-leucine-rich repeat (NBS-LRR) resistance genes and pathogenesis-related (PR) genes during berry development. Transcript levels of $P R-1$ in Norton berry skin clearly increased during the ripening phase. As in other grapevines, genes of the phenylpropanoid pathway were up-regulated in Norton as the berry developed. The most noticeable was the steady increase of transcript levels of stilbene synthase genes. Transcriptional patterns of six MYB transcription factors and eleven structural genes of the flavonoid pathway and profiles of anthocyanins and proanthocyanidins (PAs) during berry skin development were analyzed comparatively in Norton and Cabernet Sauvignon. Transcriptional patterns of MYB5A and MYB5B were similar during berry development between the two varieties, but those of MYBPA1 and MYBPA2 were strikingly different, demonstrating that the general flavonoid pathways are regulated under different MYB factors. The data showed that there were higher transcript levels of the genes encoding flavonoid-3'-O-hydroxylase $\left(F 3^{\prime} H\right)$, flavonoid-3',5'-hydroxylase $\left(F 3^{\prime}{ }^{\prime} H\right)$, leucoanthocyanidin dioxygenase (LDOX), UDP-glucose:flavonoid 3'-O-glucosyltransferase (UFGT), anthocyanidin reductase (ANR), leucoanthocyanidin reductase (LAR) 1 and LAR2 in berry skin of Norton than in those of Cabernet Sauvignon. It was also found that the total amount of anthocyanins was markedly higher in Norton than in Cabernet Sauvignon berry skin at harvest, and five anthocyanin derivatives and three PA compounds exhibited distinctive accumulation patterns in Norton berry skin.
\end{abstract}

Conclusions: This study provides an overview of the transcriptome changes and the flavonoid profiles in the berry skin of Norton, an important North American wine grape, during berry development. The steady increase of transcripts of $P R-1$ and stilbene synthase genes likely contributes to the developmentally regulated resistance during ripening of Norton berries. More studies are required to address the precise role of each stilbene synthase

\footnotetext{
* Correspondence: WenpingQiu@missouristate.edu

${ }^{1}$ Center for Grapevine Biotechnology, William H. Darr School of Agriculture,

Missouri State University, Mountain Grove, MO 65711, USA

Full list of author information is available at the end of the article
} 
gene in berry development and disease resistance. Transcriptional regulation of MYBA1, MYBA2, MYB5A and MYBPA1 as well as expression levels of their putative targets $F 3^{\prime} H, F 3^{\prime} 5^{\prime} H, L D O X, U F G T, A N R, L A R 1$, and LAR2 are highly correlated with the characteristic anthocyanin and PA profiles in Norton berry skin. These results reveal a unique pattern of the regulation of transcription and biosynthesis pathways underlying the viticultural and enological characteristics of Norton grape, and yield new insights into the understanding of the flavonoid pathway in nonvinifera grape varieties.

\section{Background}

Berry development in grapes is a complex process of physiological and biochemical changes [1]. It is initiated by hormonal signals generated after pollination [2]. The nature and origin of the hormonal signals that influence the complex processes of berry development have not been fully understood, but abscisic acid, brassinosteroids and ethylene have been implicated in these processes $[3,4]$. Although ethylene is present at the beginning of ripening, it does not show a rapid increase in concentration, and no burst of respiration occurs in grape berries [5]. Thus, grapes are non-climacteric fruits.

The berry development of grape follows a doublesigmoid pattern that is characterized by two growth phases interrupted by a lag phase (véraison) which marks the transition from herbaceous development to ripening [6]. High-throughput profiling of transcripts by using the first generation Affymetrix Vitis GeneChip has provided a comprehensive picture of gene regulation that depicts the complex biochemical pathways during berry development of $V$. vinifera grapevines $[7,8]$. The transcriptome analysis has also identified distinct transcriptional patterns and tissue-specific genes in seed, skin and pulp of grape berry [9]. The results of these studies have offered the insights into how key regulatory circuits orchestrate berry development and influence unique berry characteristics in $V$. vinifera varieties.

The skin of grape berries serves as a physical and biochemical barrier that protects ripening berries from being attacked by pathogens. During the first growth phase, the skin accumulates high levels of proanthocyanidins (PAs). The astringent properties of PAs may play a role in repelling herbivores from consuming berries before seeds are mature, and also in the protection of plants against fungal pathogens [10]. At véraison, the skin begins to accumulate anthocyanins which are the predominant pigments of grape berries. The dark color is believed to attract herbivorous animals to promote the dissemination of seeds into new territories. Supporting this proposition is the fact that the skin color of wild Vitis species berries is black. In addition to PAs and anthocyanins, the skin also accumulates flavan-3-ol monomers, although the majority of flavan-3-ols are synthesized in the grape seed [11]. The endo- and mesocarp of the berry contain large quantities of acids, primarily malic and tartaric acids, during the first growth phase, and sugars during the second growth phase of berry development [1,2].

Prior to maturity, the skin's resistance against pathogens increases in order to protect the ripening grape berries [12-14]. The high levels of flavonoid compounds in the skin are thought to contribute to the enhanced disease resistance of mature berries. It was discovered that many highly expressed genes in the skin of Cabernet Sauvignon are associated with pathogen resistance and flavonoid biosynthesis [9]. The transcriptional profiles of skin-specific genes, which were also corroborated by proteomics analysis, indicated that a set of enzymes in the anthocyanin biosynthesis pathway were significantly over-expressed in the skin of fully ripe berries [15]. A set of pathogenesis-related $(P R)$ genes, such as $P R-1, P R-2$, $P R-3, P R-4$ and $P R-5$, all increased in the ripening berry of Cabernet Sauvignon, with $P R-3$ and $P R-5$ having the most dramatic increase $[7,16]$. During véraison, the berry experiences a burst of reactive oxygen species (ROS) and a surge in the expression of genes that encode enzymes involved in the generation of antioxidants [8]. Generation of ROS is closely associated with cell death and plant defense responses [17]. The timing of accumulation of these defense-related proteins is synchronized with the initiation of the ripening berry's ability to prevent infection by pathogens [18]. There is experimental evidence that the increased expression of defense-related genes forms a protective layer in the berry skin against pathogens $[19,15]$. This supports the hypothesis that there is a correlation between the increased expression of defenserelated genes and the enhanced resistance against pathogens in the ripening berry.

The composition, conjugation and quantity of anthocyanins in red varieties determine the color density and hue of the berry skin. Anthocyanins and PAs contribute to the astringency of wine and are also antioxidants with beneficial effects on human health [20]. Transcriptional regulation of the flavonoid pathway genes has been investigated mostly in $V$. vinifera varieties. Six MYB transcription factors (MYBA1, MYBA2, MYB5A, MYB5B, MYBPA1 and MYBPA2) are associated with the regulation of the structural genes in the flavonoid pathway. MYBA1 and MYBA2 play roles in the biosynthesis of anthocyanins by activating the promoter of UFGT 
[21-23], which catalyzes the last step of anthocyanin synthesis. MYB5A and MYB5B are involved in regulating several flavonoid biosynthesis steps [24]. MYBPA1and MYBPA2 regulate the last steps of pathways in the production of PAs [22,25].

Norton is considered a $V$. aestivalis-derived variety which produces high quality red wine that is comparable to wines made from $V$. vinifera grapes. Norton leaves accumulate high levels of salicylic acid (SA) and SAassociated defense genes in comparison with Cabernet Sauvignon. Abundant SA and high expression of SAassociated defense genes may equip Norton grape with a robust innate defense system against pathogens [26]. Furthermore, total amounts of anthocyanin and phenolic acid contents are significantly higher in Norton berries than in those of $V$. vinifera $[27,28]$. Similarly to other grape varieties that originate in North America, Norton berries develop exceptionally high levels of disease resistance, which enable viticulturists to grow this grape with minimal application of pesticides in regions with high disease pressure. Transcriptomics, proteomics, and metabolic profiles of berry development of $V$. vinifera varieties Cabernet Sauvignon and Pinot Noir have been studied and documented using Affymetrix GeneChips $[7,8,15,29]$. Consequently, the synthesis of flavonoids in the berry skin, and the expression and regulation of the underlying genes are well understood in $V$. vinifera. Little is known, however, about the regulation of the biosynthesis of flavonoid compounds in the berry skin of Norton. In this study, we analyzed the transcriptional profiles of over twenty thousand genes in Norton berry skin across six developmental stages using the second generation of Affymetrix Vitis microarrays (GRAPEGEN GenChip) [30]. We discovered a high coordination between the transcriptional regulation of key transcription factors and structural genes in the flavonoid biosynthesis pathway and the accumulation profiles of flavonoid compounds. Comparative analysis of key genes in flavonoid biosynthesis and of the main flavonoid compounds between Norton and Cabernet Sauvignon revealed variety-specific patterns of gene regulation and compound biosynthesis. The results from this study yield new knowledge on the distinct chemistry and characteristics of Norton grapes.

\section{Results and Discussion}

Discovery of differentially expressed genes during Norton berry skin development

Similarly to the berry development of $V$. vinifera varieties, the development of Norton berries is characterized by a two-stage growth pattern. Sugar accumulation began at the early stages and accelerated during véraison. Also following the pattern of $V$. vinifera berry development, the levels of titratable acidity dropped at stage 34 (at 66 days after bloom $[\mathrm{DAB}]$ ) and continued to decrease until the berry was ripe. The descriptors of berry development, including berry diameter, titratable acidity and soluble solids, are presented in an accompanying paper (Ali et al., in preparation). We started sampling on June 26, 2008 when the skin could be separated from the pulp of the berry. At this point, the berry was at stage 31 (17 DAB) on the Eichorn-Lorenz phenological scale. Subsequently, skin samples were taken at stages 33, 34, 35, 36, 37 and 38, corresponding to $37,66,71$ (véraison), 85, 99, and 127 DAB. Skin tissue was frozen in liquid nitrogen and total RNA was extracted subsequently. The RNA was then labeled and hybridized to GRAPEGEN Affymetrix GeneChips. Processing of raw intensity values in CEL files and subsequent normalization and Median polishing were described in the paper (Ali et al., in preparation).

A Principal Component Analysis (PCA) of the eighteen arrays was performed to assess the similarity of expression values among the replicates (Additional File 1). The results from the PCA indicated a high degree of similarity among three biological replicates that were clustered tightly within the scatterplot. In addition, PCA showed that data of two proximal developmental stages were more similar to each other than data of distal developmental stages. There is a clear alignment and separation of developmental stages along the $\mathrm{PC} 1$ in the plot (Additional File 1). The eighteen sets of the data were then converted to $\mathrm{z}$-scores and subjected to two-way unsupervised agglomerative cluster analysis (Additional File 2). This analysis showed that each stage represents a major branch which contains only the three biological replicate data for that stage. The results from these two analyses demonstrated that there is a good reproducibility among the three biological replicates and thus all data were included in the analysis. Pearson correlation coefficients between biological replicates were also calculated and were in the range of 0.9812 to 0.9976 (Additional File 3), further corroborating significant correlations between biological replicates in each developmental stage.

After the data of all eighteen arrays were processed and assessed for quality, the error-weighted intensity experiment definitions (EDs) were calculated by averaging the intensity of three biological replicates for each stage and then error-corrected using the Rosetta error model [31]. ANOVA was conducted on the error-weighted intensity of three biological replicates at each stage across six developmental stages with the Benjamini-Hochberg False Discovery Rate multiple test correction [32]. This resulted in the discovery of 15,823 probe sets that exhibited significant variations at the transcript levels between at least two developmental stages at $P \leq 0.001$ (Additional File 4). The differentially expressed probe sets comprise more 
than $78 \%$ of all probe sets on the microarray, indicating that a large number of genes represented on the array changed significantly at transcript levels at some points during berry development. To discover the genes whose transcript levels varied significantly from a baseline calculated from all six developmental stages, the intensity EDs of each probe set were divided by an error-weighted average of all six developmental stages. Under the criteria of absolute fold-change $\geq 2.0$ in at least one developmental stage and having a LogRatio $P$-value $\leq 0.001$ in at least one stage, we identified 3,352 probe sets (Additional File 5 ). We selected this group of the most significantly expressed genes for the subsequent analysis. The large number of transcripts that changed at expression levels corroborated earlier findings that genes of different functions were detected in the berry skin at the beginning of véraison and the later stages of ripening, reflecting the dramatic biochemical changes that take place during berry ripening $[7,15]$.

\section{Cluster analysis of differentially expressed genes in Norton berry skin}

We used the nucleotide sequence from which each set of probes was designed to acquire the best-matched GSVIVT ID in Genoscope (http://www.genoscope.cns.fr/ externe/GenomeBrowser/Vitis/) or TC number in DFCI Grape Gene Index (http://compbio.dfci.harvard.edu/tgi/ cgi-bin/tgi/gimain.pl?gudb=grape). The total of 3,352 probe sets represented 2,760 unique genes. We removed those probe sets where more than one probe set was assigned to the same GSVIVT ID or TC numbers but showed different expression patterns, and compiled them into a separate file for future analysis. At this time, it is not possible to discern what factors, such as alternatively spliced transcripts or degradation biases of the 5'-end and 3'-end portion of mRNA, influence the expression levels of these genes. We subjected the $\log _{2}-$ transformed fold-change of the remaining 2,359 unigenes to clustering by the $k$-means method. A total of 20 clusters were defined from this group of genes based on the figure of merit value (Additional File 6).

Transcript abundance of these genes in cluster 1, 12, 13, 18 and 20 increased after véraison (Figure 1). These five clusters contained a total of 1,053 genes. Cluster 11 (113 genes) and Cluster 16 (42 genes) represented a pattern of transient increase and decrease, respectively, of transcript levels at the onset of véraison and subsequently unchanged post-véraison. The expression pattern of cluster 8 (65 genes) and cluster 19 (60 genes) was reciprocal. In cluster 8 , transcript levels increased pre-véraison and decreased post-véraison. In cluster 19, transcript levels decreased at véraison, but increased both pre-véraison and post-véraison. The remaining eleven clusters included 1,026 genes and exhibited a pattern of steady decline post-véraison. The genes in each cluster are listed in Additional File 6.

\section{Developmental regulation of defense-related genes}

A total of 48 differentially expressed genes were associated with defense, disease resistance, and hypersensitive response (Table 1). Among them, twenty one genes were up-regulated, and twenty five genes were down-regulated post-véraison. These defense-related genes include the well characterized polygalacturonase inhibiting protein (PGIP), dirigent protein, NBS-LRR, Non-race-specific disease resistance 1 (NDR1), powdery mildew resistant 5 (PMR5), and harpin-induced protein 1 genes.

Especially noticeable is the expression profile of the $P R-1$ gene, which is an indicator for the induction of local defense and systemic acquired resistance (SAR) in plants $[33,34]$. In grapevine, the $P R-1$ gene (GSVIVT00038581001) was induced by salicylic acid [35], and up-regulated after infection with the powdery mildew (PM) fungal pathogen Erysiphe necator [26]. Transcript levels of $P R-1$ increased progressively postvéraison in both Norton (cluster 18, Figure 1 and Table 1), and Cabernet Sauvignon [7,29]. The gene AtWRKY75 plays an important role in the activation of basal and resistance $(R)$ gene-mediated resistance in Arabidopsis [36], and transcript levels of its grapevine ortholog increased in response to PM infection [26]. Interestingly, the grapevine WRKY75 ortholog was discovered in cluster 18. Four NBS-LRR genes were also identified in cluster 18, indicating these proteins are regulated developmentally in grape (Table 1 ). Plant NBS-LRR proteins are receptors that directly or indirectly recognize pathogen-deployed proteins, and this specific recognition triggers plant defense responses $[37,38]$. In some cases, they also play a role in the regulation of developmental pathways [39].

Five probe sets were annotated as thaumatin-like proteins and two as osmotins. Their transcript levels increased significantly in the late stages of Norton berry development (Additional File 5 and 6), as was shown previously in varieties of $V$. vinifera $[7,29]$. Thaumatinlike proteins inhibit spore germination and hyphal growth of E. necator, Phomopsis viticola, and Botrytis cinerea [40]. We found that transcript levels of five chitinase genes increased post-véraison in Norton berry skin (cluster 12, 13, 19, and 20). Transcript levels of basic class I (VCHIT1b) and a class III (VCH3) chitinase of grapevines increase in response to the chemical activators of SAR and are considered as markers of SAR [41]. Furthermore, enzymatic activities of chitinase and $3-1,3-$ glucanase also increase during berry development in the absence of pathogens [15]. Non-specific lipid transfer proteins (nsLTPs) belong to a family of small cystein-rich 


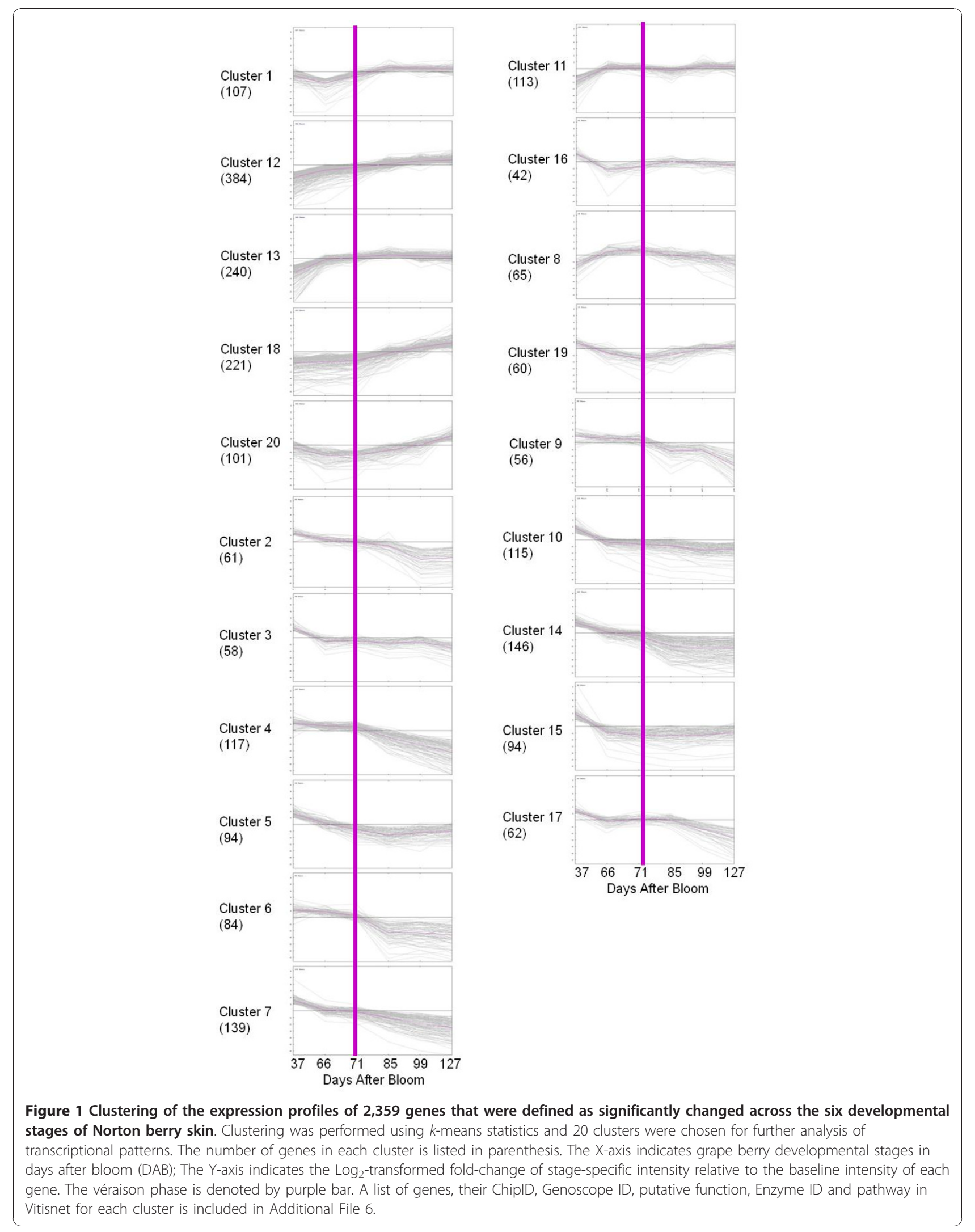


Table 1 Transcriptional profiles of genes in Norton berry skin that are associated with defense pathways

\begin{tabular}{|c|c|c|c|c|c|}
\hline Cluster $^{A}$ & Affymetrix ChipID & Genoscope ID & Function (VitisNet) ${ }^{\mathrm{B}}$ & KEGG & Pathway (VitisNet) \\
\hline \multicolumn{6}{|c|}{ Up-regulation post véraison } \\
\hline 1 & WTU11871_s_at & GSVIVT00025506001 & Polygalacturonase inhibiting protein PGIP1 & PGIP & Defense \\
\hline 12 & WTU6661_at & $\overline{\text { GSVIVT00005104001 }}$ & Dirigent & & Defense \\
\hline 18 & WTU13759_at & $\overline{\text { GSVIVT00038581001 }}$ & Pathogenesis-related protein 1 & PRP1 & Defense \\
\hline 18 & WTU1755_at & GSVIVT00033081001 & Pathogenesis protein 10.1 & & Defense \\
\hline 18 & WTU39372_at & GSVIVT00024739001 & Dirigent protein & & Defense \\
\hline 18 & WTU21514_x_at & GSVIVT00024741001 & Dirigent protein & & Defense \\
\hline 18 & WTU8656_at & GSVIVT00036870001 & Epoxide hydrolase 2 & 3.3.2.10 & Defense \\
\hline 13 & WTU10916_at & $\overline{\text { GSVIVT00018587001 }}$ & Ripening induced protein & & Defense response \\
\hline 20 & WTU4789_at & GSVIVT00007703001 & NtPRp27 secretory protein & & Defense response \\
\hline 1 & WTU10868_at & $\overline{\text { GSVIVT00037825001 }}$ & Disease resistance protein & & Disease resistance \\
\hline 18 & WTU16881_at & GSVIVT00028656001 & Disease resistance protein (NBS-LRR class) & & Disease resistance \\
\hline 20 & WTU7497_s_at & $\overline{\text { GSVIVT00000261001 }}$ & Disease resistance protein (TIR-NBS class) & & Disease resistance \\
\hline 20 & WTU36452_at & $\overline{\text { GSVIVT00038332001 }}$ & TIR-NBS-LRR disease resistance & & Disease resistance \\
\hline 12 & WTU40849_s_at & $\overline{\text { GSVIVTT00030517001 }}$ & Major latex protein 22 & & Disease resistance \\
\hline 12 & WTU35326_at & GSVIVT00002134001 & Seed maturation protein PM41 & & Disease resistance \\
\hline 13 & WTU2601_at & $\overline{\text { GSVIVT00018817001 }}$ & PMR5 (POWDERY MILDEW RESISTANT 5) & & Disease resistance \\
\hline 20 & WTU9483_at & GSVIVT00000260001 & TIR-NBS-LRR-TIR disease resistance protein & & Disease resistance \\
\hline 20 & WTU2928_at & GSVIVT00021517001 & Hairpin inducing protein 1-like 9 & & Hypersensitive response \\
\hline 20 & WTU37592_at & $\overline{\text { GSVIVT00023399001 }}$ & Hairpin induced protein & & Hypersensitive response \\
\hline 18 & WTU11329_at & GSVIVT00030027001 & SP1L1 (SPIRAL1-LIKE1) & & Pathogen \\
\hline 18 & WTU1632_at & $\overline{\text { GSVIVT00030524001 }}$ & Bet $v$ I allergen & & Pathogenesis \\
\hline \multicolumn{6}{|c|}{ Up-down-up regulation } \\
\hline 19 & WTU4500_s_at & GSVIVT00036464001 & Viral-response family protein-like & & Defense \\
\hline 19 & WTU7944_at & $\overline{\text { GSVIVT00016484001 }}$ & BREVIS RADIX 4 & & Disease resistance \\
\hline \multicolumn{6}{|c|}{ Down-regulation post véraison } \\
\hline 9 & WTU3745_s_at & GSVIVT00024648001 & Polygalacturonase inhibitor protein & PGIP & Defense \\
\hline 7 & WTU3256_at & GSVIVT00024747001 & Dirigent protein pDIR9 & & Defense \\
\hline 14 & WTU4542_at & GSVIVT00016676001 & Lachrymatory factor synthase & & Defense \\
\hline 15 & WTU28352_at & GSVIVT00024745001 & Dirigent protein & & Defense \\
\hline 14 & WTU2350_at & $\overline{\text { GSVIVT00033031001 }}$ & Epoxide hydrolase & 3.3.2.10 & Defense \\
\hline 17 & WTU2606_at & GSVIVT00025834001 & Epoxide hydrolase 2 & 3.3.2.10 & Defense \\
\hline 3 & WTU34452_at & $\overline{\text { GSVIVT00004842001 }}$ & Disease resistance protein (TIR-NBS-LRR class) & & Disease resistance \\
\hline 5 & WTU2751_s_at & $\overline{\text { GSVIVT00033825001 }}$ & Disease resistance protein & & Disease resistance \\
\hline 7 & WTU20455_at & $\overline{\text { GSVIVT00018767001 }}$ & Receptor kinase TRKa & & Disease resistance \\
\hline 7 & WTU21216_at & $\overline{\text { GSVIVT00020681001 }}$ & Disease resistance protein (NBS-LRR class) & & Disease resistance \\
\hline 14 & WTU10907_at & $\overline{\text { GSVIVT00011855001 }}$ & Hervf1 protein & & Disease resistance \\
\hline 14 & WTU1732_at & $\overline{\text { GSVIVT00025424001 }}$ & Disease resistance responsive & & Disease resistance \\
\hline 14 & WTU34204_s_at & $\overline{\text { GSVIVT00025429001 }}$ & Disease resistance responsive & & Disease resistance \\
\hline 15 & WTU24464_at & GSVIVT00026768001 & Disease resistance protein (CC-NBS-LRR class) & & Disease resistance \\
\hline 2 & WTU52_at & $\overline{\text { GSVIVT00027396001 }}$ & NDR1 (NON RACE-SPECIFIC DISEASE RESISTANCE) & & Disease resistance \\
\hline 3 & WTU8917_at & $\overline{\text { GSVIVT00033069001 }}$ & Major allergen Pru ar 1 & & Disease resistance \\
\hline 5 & WTU29478_at & GSVIVT00025399001 & PMR5 (POWDERY MILDEW RESISTANT 5) & & Disease resistance \\
\hline 9 & WTU5508_s_at & $\overline{\text { GSVIVT00033067001 }}$ & Major cherry allergen Pru av 1.0202 & & Disease resistance \\
\hline 14 & WTU30737_at & $\overline{\text { GSVIVT00018816001 }}$ & PMR5 (POWDERY MILDEW RESISTANT 5) & & Disease resistance \\
\hline 3 & WTU2005_at & $\overline{\text { GSVIVT00026172001 }}$ & Hairpin induced 1 & & Hypersensitive response \\
\hline 5 & WTU10307_x_at & GSVIVT00006738001 & Hairpin induced 1 & & Hypersensitive response \\
\hline 14 & WTU14941_at & GSVIVT00034176001 & Hairpin induced 1 & & Hypersensitive response \\
\hline 15 & WTU16087_at & $\overline{\text { GSVIVT00032401001 }}$ & G protein protein gamma subunit (AGG2) & & Pathogen defense \\
\hline 17 & WTU27983_at & GSVIVT00023169001 & Mlo3 & K08472 & Pathogen defense \\
\hline 17 & WTU7548_x_at & $\overline{\text { GSVIVT00030529001 }}$ & Bet $v$ I allergen & & Pathogenesis \\
\hline
\end{tabular}

A Expression profiling of each cluster is shown in Figure 1. ${ }^{\mathrm{B}}$ Function annotation and pathway assignment of each gene were based on VitisNet (http:// vitis-dormancy.sdstate.org/pathways.cfm) 
proteins that are induced in response to fungal elicitors and are associated with grapevine defense [42-44]. A possible LTP-jasmonic acid complex may protect grape berries against B. cinerea [42]. Transcripts of one probe set (GSVIVT00037486001) encoding VvLPT1, which are more prevalent in berry skin than in seeds [9], also increased steadily in Norton berries post-véraison (Cluster 1, Figure 1 and Additional File 6). In summary, differential expression of these defense-related genes indicates a developmentally regulated modulation of defense responses during ripening in Norton berry skin.

\section{Transcripts of stilbene synthase genes increased in Norton berry skin post-véraison}

The cis- and trans-piceid compounds of the stilbene family constitute a major group of phytoalexins in grapevines that are involved in the defense responses to pathogens [45]. They have been shown to have antifungal activities against several fungal pathogens including Plasmopara viticola [46] and B. cinerea $[47,48]$. They also exhibit antibacterial activity against Xylella fastidiosa [49], the pathogen of Pierce's disease on grapevine. In addition, stilbenic compounds possess anticancer and anti-inflammatory activities that have potential benefits to human health [50]. Stilbene synthase (STS) is the key enzyme that catalyzes the formation of 3', 4', 5'-trihydroxystilbene (resveratrol) via the condensation of one 4-coumaroyl-CoA and three malonyl-CoA molecules (Figure 2A). This condensation reaction represents a branch point in the phenylpropanoid pathway, at which CHS channels 4-coumaroyl-CoA molecules towards flavonoid synthesis and STS towards stilbene synthesis.

Grape berry skin is the main tissue where the synthesis of stilbenes occurs [51]. STS was found to be localized mostly in the cell wall of hypodermal cells in the exocarp, which is in agreement with the detection of stilbenic compounds mainly in the exocarp during berry development [51]. It was also demonstrated that stilbenic compounds and transcripts of the key genes $P A L, 4 C L$, and $S T S$ accumulated progressively in ripening berries of Pinot Noir [52] and Corvina [53]. The composition of stilbenic compounds differs significantly among grape varieties. Mature berries of Pinot Noir contain the highest levels of stilbenes, while the stilbene content of Cabernet Sauvignon berries is ranked 41st among 48 redskinned grapes [52]. There is a high correlation between the transcript levels of $P A L, 4 C L$, and STS and the abundance of stilbenic compounds in grape varieties [52,53]. We found that six of the ten paralogous STS genes on the GrapeGen Chip are grouped into clusters 18 and 20, and the transcripts of these genes increased steadily and significantly post-véraison (Figure 1). Interestingly, $P A L$ and $4 C L$ were also found in clusters 18 and 20, in which transcripts of these genes significantly increased in the final two stages (Figure 1). Highly coordinated expression of $P A L, 4 C L$, and $S T S$ post-véraison strongly supports the conclusion that the stilbene biosynthesis pathway is upregulated during the development of Norton berry skin. In our previous microarray analysis of the pathogeninduced transcriptome in grapevines, we discovered that STS genes were strongly induced in response to PM infection [26]. These results confirm that stilbenes, together with other phytoalexins and defense-related proteins, are part of the defense weaponry for protecting berries from pathogen attacks. This defense strategy appears to be developmentally regulated in Norton berry skin.

\section{Coordinated expression of the phenylpropanoid and flavonoid pathways}

Results of previous microarray analyses of tissue-specific transcriptomes demonstrated that the majority of genes encoding enzymes in the biosynthesis of flavonoids, lignin, anthocyanins and proanthocyanidins were expressed preferentially in the berry skin of grapevine [9]. These genes include $P A L, C 4 H$, and $4 C L$, encoding key enzymes which catalyze the first three steps of the phenylpropanoid pathway (Figure $2 \mathrm{~A}$ ). The present microarray analysis also showed that transcripts of three $P A L$ genes and one $4 C L$ gene increased significantly in Norton berry skin post-véraison (Table 2). The increasing levels of $P A L$ and $4 C L$ transcripts most likely led to higher accumulation of the substrate 4-coumaryl-CoA for the down-stream pathways. This trend coordinates well with the transcriptional regulation of chalcone synthase (CHS) (GSVIVT00037967001), six STSs, DFR (GSVIVT00014584001) and GSVIVT 00036313001), LDOX (GSVIVT00001063001), and UFGT (GSVIVT00014047001). Transcripts of these genes increased post-véraison (Table 2). This up-regulation of the phenylpropanoid pathway in the skin of the ripening berry has also been observed in Cabernet Sauvignon [15]. Interestingly, the genes that were expressed at the highest level in Cabernet Sauvignon encoded enzymes mostly in the flavonoid biosynthesis pathway downstream of PAL, $\mathrm{C} 4 \mathrm{H}$ and $4 \mathrm{CL}$.

After we had compared the previous microarray analysis of Cabernet Sauvignon berry development [7] with the present results in Norton (Table 2), we discovered that the two grape varieties share eight genes that are differentially expressed in the flavonoid pathway. Particularly interesting is the finding that transcripts of F3H (GSVIVT00036784001), flavonol synthase (FLS) (GSVIVT00015347001), and CHS (GSVIVT00037967001) decreased progressively during Cabernet Sauvignon berry development, but increased steadily in Norton. 
A

General phenylpropanoid pathway

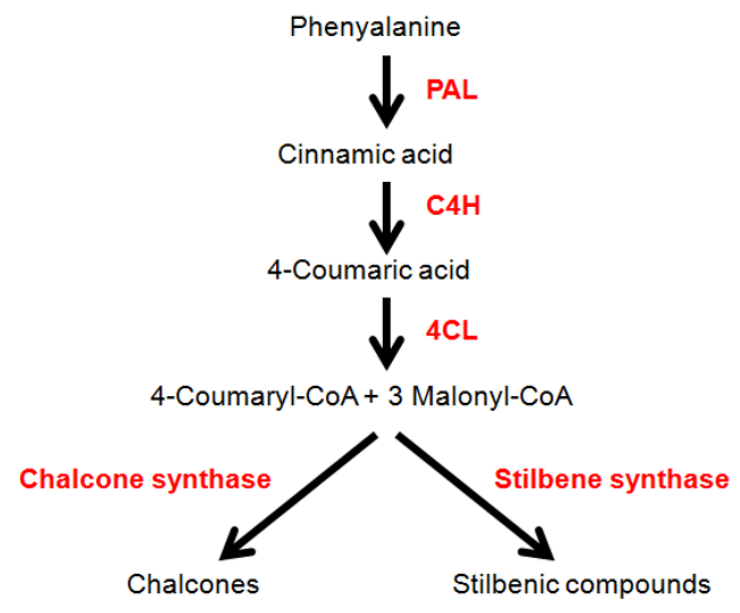

B

Flavonoid Biosynthesis Pathway

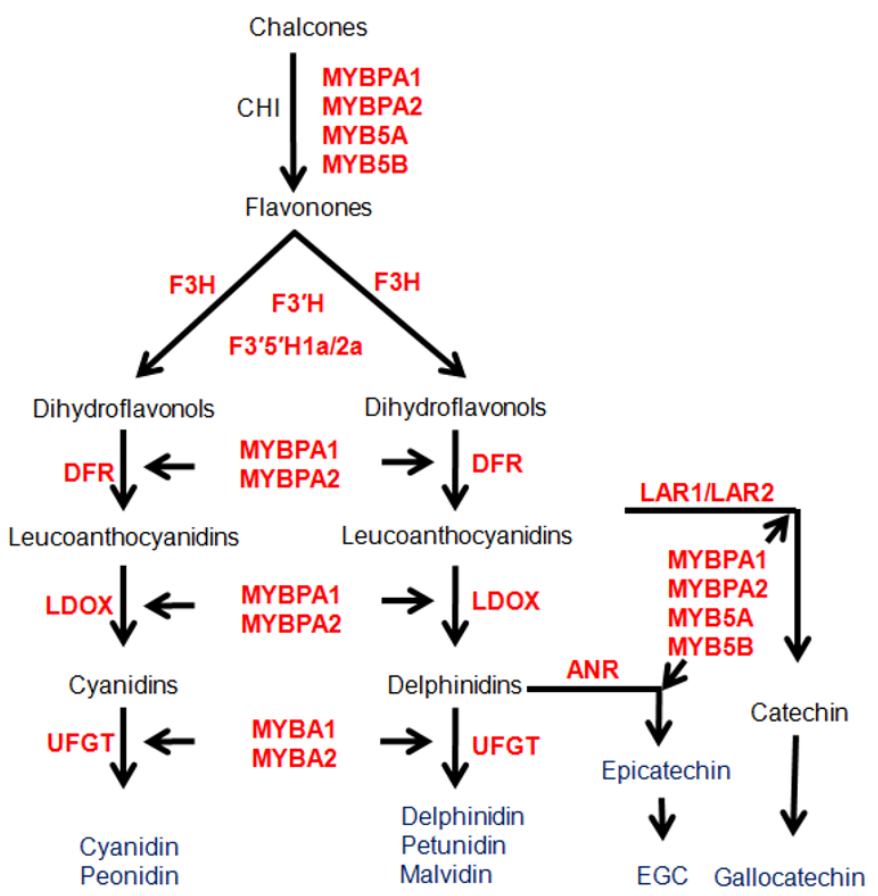

Figure 2 Overview of the general phenylpropanoid pathway. A: A simplified representation of the phenylpropanoid pathway leading to the production of chalcones and stilbenic compounds; B: The flavonoid biosynthesis pathway that leads to the production of anthocyanins and proanthocyanidins; six MYB transcription factors are indicated along the branches that are likely involved in the transcriptional regulation of the structural genes. PAL, phenylalanine ammonia-lyase; $\mathrm{C} 4 \mathrm{H}$, cinnamate 4-hydroxylase; $4 \mathrm{CL}$, 4-coumarate-CoA ligase; $\mathrm{CH}$, chalcone isomerase; $\mathrm{F3H}$, flavanone 3-hydroxylase; F3'H, flavonoid-3'-O-hydroxylase; F3'5'H, flavonoid-3',5'-hydroxylase; DFR, dihydroflavonol-4-reductase; LDOX,

leucoanthocyanidin dioxygenase; UFGT, UDP-glucose:flavonoid-3-O-glucosyltransferase; ANR, anthocyanidin reductase; LAR, leucoanthocyanidin reductase; EGC, epigallocatechin. 
Table 2 Transcriptional profiles of genes in Norton berry skin that are associated with secondary metabolism

\begin{tabular}{|c|c|c|c|c|c|}
\hline Cluster $^{\mathrm{A}}$ & Affymetrix ChipID & Genoscope ID & Function (VitisNet) $^{\mathrm{B}}$ & KEGG & Pathway (VitisNet) \\
\hline 1 & VVTU703_s_at & GSVIVT00018175001 & Phenylalanine ammonia lyase 2 (PAL2) & 4.3.1.5 & Phenylpropanoid \\
\hline 1 & VVTU12705_s_at & GSVIVT00024561001 & Phenylalanine ammonia lyase (PAL) & 4.3.1.5 & Phenylpropanoid \\
\hline 18 & VVTU26285_at & GSVIVT00013936001 & Phenylalanine ammonia lyase (PAL) & 4.3.1.5 & Phenylpropanoid \\
\hline 4 & WTU39693_at & GSVIVT00008924001 & Cinnamyl alcohol dehydrogenase (CAD) & 1.1.1.195 & Phenylpropanoid \\
\hline 6 & WTU2766_at & GSVIVT00011484001 & Sinapyl alcohol dehydrogenase (SAD) & 1.1.1.195 & Phenylpropanoid \\
\hline 10 & WTU14855_at & GSVIVT00024588001 & Cinnamyl alcohol dehydrogenase (CAD) & 1.1.1.195 & Phenylpropanoid \\
\hline 20 & VVTU21888_at & GSVIVT00011639001 & Cinnamyl alcohol dehydrogenase (CAD) & 1.1.1.195 & Phenylpropanoid \\
\hline 2 & WTU13147_s_at & GSVIVT00013987001 & Cinnamoyl-CoA reductase (CCR) & 1.2.1.44 & Phenylpropanoid \\
\hline 7 & WTU12930_s_at & GSVIVT00033763001 & Cinnamoyl-CoA reductase (CCR) & 1.2.1.44 & Phenylpropanoid \\
\hline 12 & VVTU3517_at & GSVIVT00015738001 & Cinnamoyl-CoA reductase (CCR) & 1.2.1.44 & Phenylpropanoid \\
\hline 13 & VVTU914_at & GSVIVT00038153001 & Cinnamoyl-CoA reductase (CCR) & 1.2.1.44 & Phenylpropanoid \\
\hline 20 & VVTU15680_at & GSVIVT00020726001 & Cinnamoyl-CoA reductase (CCR) & 1.2.1.44 & Phenylpropanoid \\
\hline 13 & VVTU4884_at & GSVIVT00002825001 & Caffeoyl-CoA O-methyltransferase (CCoAOMT) & 2.1.1.104 & Phenylpropanoid \\
\hline 18 & VVTU36108_at & GSVIVT00025990001 & Caffeic acid O-methyltransferase (CAOMT) & 2.1 .1 .68 & Phenylpropanoid \\
\hline 18 & VVTU6966_s_at & GSVIVT00026179001 & Caffeate 3-O-methyltransferase 1 (COMT) & 2.1.1.68 & Phenylpropanoid \\
\hline 12 & VVTU34546_at & GSVIVT00009234001 & Stilbene synthase (STS) & 2.3.1.95 & Phenylpropanoid \\
\hline 18 & VVTU34913_at & GSVIVT00007353001 & Stilbene synthase (STS) & 2.3.1.95 & Phenylpropanoid \\
\hline 18 & VVTU34551_x_at & GSVIVT00031875001 & Stilbene synthase (STS) & 2.3.1.95 & Phenylpropanoid \\
\hline 18 & VVTU11765_at & GSVIVT00004049001 & Stilbene synthase (STS) & 2.3.1.95 & Phenylpropanoid \\
\hline 18 & VVTU7619_x_at & GSVIVT00005196001 & Stilbene synthase (STS) & 2.3.1.95 & Phenylpropanoid \\
\hline 18 & VVTU2775_x_at & GSVIVT00007358001 & Stilbene synthase (STS) & 2.3.1.95 & Phenylpropanoid \\
\hline 18 & VVTU18886_x_at & GSVIVT00007364001 & Stilbene synthase (STS) & 2.3.1.95 & Phenylpropanoid \\
\hline 18 & VVTU6035_x_at & GSVIVT00009221001 & Stilbene synthase (STS) & 2.3.1.95 & Phenylpropanoid \\
\hline 20 & VVTU26310_s_at & GSVIVT00031885001 & Stilbene synthase (STS) & 2.3.1.95 & Phenylpropanoid \\
\hline 20 & VVTU2671_at & GSVIVT00009225001 & Stilbene synthase (STS) & 2.3.1.95 & Phenylpropanoid \\
\hline 7 & WTU15752_at & GSVIVT00002505001 & Pinoresinol forming dirigent protein & DIRPR & Phenylpropanoid \\
\hline 16 & WTU8264_at & GSVIVT00023306001 & p-Coumaroyl shikimate 3'-hydroxylase isoform 1 & K09754 & Phenylpropanoid \\
\hline 14 & WTU25372_at & GSVIVT00017649001 & Ferulate 5-hydroxylase (F5H) & K09755 & Phenylpropanoid \\
\hline 18 & VVTU8974_at & GSVIVT00036840001 & Ferulate 5-hydroxylase ( $\mathrm{F} 5 \mathrm{H})$ & K09755 & Phenylpropanoid \\
\hline 14 & WTU34012_at & GSVIVT00017653001 & Ferulate 5-hydroxylase (F5H) & K09755 & Phenylpropanoid \\
\hline 2 & WTU6513_s_at & GSVIVT00038750001 & Pinoresinol-lariciresinol reductase & $P L R$ & Phenylpropanoid \\
\hline 15 & WTU15529_s_at & GSVIVT00021542001 & Secoisolariciresinol dehydrogenase & SIRD & Phenylpropanoid \\
\hline 20 & VVTU2645_at & GSVIVT00031383001 & 4-Coumarate-CoA ligase 2 (4CL) & 6.2 .1 .12 & Phenylpropanoid \\
\hline 1 & VVTU17924_s_at* & GSVIVT00014584001 & Dihydroflavonol 4-reductase (DFR) & 1.1.1.219 & Flavonoid \\
\hline 12 & VVTU14294_at & GSVIVT00036313001 & Dihydroflavonol-4-reductase (DFR) & 1.1.1.219 & Flavonoid \\
\hline 13 & VVTU36178_s_at* & GSVIVT00001063001 & Leucoanthocyanidin dioxgenase (LDOX) & 1.14.11.19 & Flavonoid \\
\hline 11 & WTU9714_at & GSVIVT00007249001 & Flavonol synthase (FLS) & 1.14.11.23 & Flavonoid \\
\hline 13 & VVTU33390_s_at & GSVIVT00031249001 & Flavonol synthase (FLS) & 1.14.11.23 & Flavonoid \\
\hline 14 & WTU13981_at & GSVIVT00007247001 & Flavonol synthase (FLS) & 1.14.11.23 & Flavonoid \\
\hline 18 & VVTU2456_s_at & GSVIVT00015347001 & Flavonol synthase (FLS) & 1.14.11.23 & Flavonoid \\
\hline 10 & WTU16387_at & GSVIVT00015842001 & Naringenin,2-oxoglutarate 3-dioxygenase & 1.14.11.9 & Flavonoid \\
\hline 13 & VVTU39787_s_at & GSVIVT00036784001 & Flavanone 3-hydroxylase (F3H) & 1.14.11.9 & Flavonoid \\
\hline 13 & VVTU37475_at & GSVIVT00037165001 & Flavanone 3-hydroxylase (F3H) & 1.14.11.9 & Flavonoid \\
\hline 1 & VVTU7778_at & GSVIVT00034070001 & Flavonoid 3-monooxygenase & 1.14.13.21 & Flavonoid \\
\hline 4 & WTU6932_at & GSVIVT00016437001 & Flavonoid 3-monooxygenase & 1.14.13.21 & Flavonoid \\
\hline 4 & WTU25410_s_at & GSVIVT00036466001 & Flavonoid 3-monooxygenase & 1.14.13.21 & Flavonoid \\
\hline 7 & WTU6362_at & GSVIVT00017654001 & Flavonoid 3-monooxygenase & 1.14.13.21 & Flavonoid \\
\hline 13 & VVTU35884_at & GSVIVT00022300001 & Flavonoid $3^{\prime}, 5^{\prime}$-hydroxylase $\left(\mathrm{F}^{\prime}{ }^{\prime}{ }^{\prime} \mathrm{H}\right)$ & 1.14.13.88 & Flavonoid \\
\hline 10 & WTU13083_at* & GSVIVT00005344001 & Anthocyanidin reductase (ANR) & 1.3.1.77 & Flavonoid \\
\hline 13 & VVTU9453_at & GSVIVT00000479001 & Quercetin 3-O-methyltransferase 1 & 2.1.1.76 & Flavonoid \\
\hline 1 & VVTU39820_s_at & GSVIVT00037967001 & Chalcone synthase(CHS) & 2.3.1.74 & Flavonoid \\
\hline
\end{tabular}


Table 2 Transcriptional profiles of genes in Norton berry skin that are associated with secondary metabolism (Continued)

\begin{tabular}{|c|c|c|c|c|c|}
\hline 5 & WTU15193_at & GSVIVT00003466001 & UDP-glucose:flavonoid 7-O-glucosyltransferase (UFGT) & 2.4.1.237 & Flavonoid \\
\hline 14 & WTU22370_at & GSVIVT00033493001 & UDP-glucose:flavonoid 7-O-glucosyltransferase (UFGT) & 2.4.1.237 & Flavonoid \\
\hline 13 & WTU17578_s_at* & $\overline{\text { GSVIVT00014047001 }}$ & UDP-glucose:flavonoid 3-O-glucosyltransferase (UFGT) & 2.4.1.91 & Flavonoid \\
\hline 3 & WTU15110_at & GSVIVT00001621001 & Flavonol 3-sulfotransferase & 2.8.2.25 & Flavonoid \\
\hline 1 & VVTU3684_s_at & GSVIVT00029440001 & Chalcone flavanone isomerase $(\mathrm{CHI})$ & 5.5.1.6 & Flavonoid \\
\hline 17 & WTU563_at & $\overline{\text { GSVIVT00020652001 }}$ & Chalcone isomerase (CHI) & 5.5.1.6 & Flavonoid \\
\hline 10 & WTU9073_x_at & GSVIVT00009968001 & UDP-glucose: anthocyanidin 5,3-O-glucosyltransferase & 2.4.1.238 & Flavonoid \\
\hline 12 & VVTU24324_at & $\overline{\text { GSVIVT00024127001 }}$ & Anthocyanidin 3-O-glucosyltransferase & 2.4.1.115 & Anthocyanin \\
\hline 18 & VVTU35521_at & $\overline{\text { GSVIVT00024993001 }}$ & Anthocyanidin 3-O-glucosyltransferase & 2.4.1.115 & Anthocyanin \\
\hline 19 & WTU15768_at & $\overline{\text { GSVIVT00037558001 }}$ & Anthocyanidin 3-O-glucosyltransferase & 2.4.1.115 & Anthocyanin \\
\hline 20 & VVTU14014_at & $\overline{\text { GSVIVT00005849001 }}$ & Anthocyanidin 3-O-glucosyltransferase & 2.4.1.115 & Anthocyanin \\
\hline 7 & WTU8698_at & GSVIVT000008206001 & Anthocyanidin rhamnosyl-transferase & RHATR & Anthocyanin \\
\hline 8 & WTU10613_at & $\overline{\text { GSVIVT00026922001 }}$ & Anthocyanidin rhamnosyl-transferase & RHATR & Anthocyanin \\
\hline 13 & VVTU7774_at & $\overline{\text { GSVIVT00011809001 }}$ & UDP-rhamnose/rhamnosyltransferase & RHATR & Anthocyanin \\
\hline 5 & WTU8944_x_at & GSVIVT00001860001 & UDP-glucose: anthocyanidin 5,3-0-glucosyltransferase & RHGT1 & Anthocyanin \\
\hline 12 & VVTU14620_at & $\overline{\text { GSVIVT00001853001 }}$ & UDP-glucose: anthocyanidin 5,3-0-glucosyltransferase & RHGT1 & Anthocyanin \\
\hline 16 & VVTU15845_at & $\overline{\text { GSVIVT00001851001 }}$ & UDP-glucose: anthocyanidin 5,3-0-glucosyltransferase & RHGT1 & Anthocyanin \\
\hline 17 & WTU15902_at & $\overline{\text { GSVIVT00001859001 }}$ & UDP-glucose: anthocyanidin 5,3-0-glucosyltransferase & RHGT1 & Anthocyanin \\
\hline 18 & VVTU36907_at & $\overline{\text { GSVIVT00024130001 }}$ & UDP-glucose: anthocyanidin 5,3-0-glucosyltransferase & RHGT1 & Anthocyanin \\
\hline 3 & WTU5076_s_at & GSVIVT00033502001 & UDP-glucoronosyl/UDP-glucosyl transferase UGT75C1 & UGT75C1 & Anthocyanin \\
\hline 15 & WTU38572_at & GSVIVT00025511001 & CYP93A1 2-hydroxyisoflavanone synthase & 1.14 .13 .86 & Isoflavonoid \\
\hline 13 & VVTU2075_at & GSVIVT00019588001 & CYP81E1 Isoflavone 2'-hydroxylase & 1.14.13.89 & Isoflavonoid \\
\hline 20 & VVTU22627_at & $\overline{\text { GSVIVT00019595001 }}$ & CYP81E1 Isoflavone 2'-hydroxylase & 1.14.13.89 & Isoflavonoid \\
\hline 4 & WTU3973_at & GSVIVT00026339001 & 2'-hydroxy isoflavone/dihydroflavonol reductase & 1.3.1.45 & Isoflavonoid \\
\hline 8 & WTU6973_at & $\overline{\text { GSVIVT00003030001 }}$ & Isoflavone methyltransferase & 2.1.1.46 & Isoflavonoid \\
\hline
\end{tabular}

A Clusters in bold exhibit steady increase of transcript abundance post véraison; Clusters in italics show decrease of transcript abundance post véraison. Expression profiling of each cluster is shown in Figure 1. ${ }^{B}$ Function annotation and pathway assignment of each gene were based on VitisNet (http:// vitis-dormancy.sdstate.org/pathways.cfm). The genes (DFR, LDOX, ANR, UFGT) with asterisk have the same GSVIVT ID and display similar expression profiling as in $\mathrm{qPCR}$.

\section{Transcription profiles of flavonoid biosynthesis genes} differ in the two varieties

The differential expression of flavonoid biosynthesis genes in Norton berry skin development prompted us to compare the transcript abundance of the most relevant genes in Norton with those in Cabernet Sauvignon. We conducted qPCR assays to compare transcript levels of eleven genes between the two varieties (Additional File 7 ). We chose these eleven genes based on their key roles in the pathway that F3'H, F3'5' $\mathrm{H}-1 \mathrm{a}$ and -2a, DFR, LDOX, and UFGT are involved in biosynthesis of anthocyanins while ANR and LAR1/2 catalyze PA synthesis (Figure 2B). Expression of the eleven genes exhibited distinctive patterns between the two varieties (Figure 3). Transcripts of F3'H, F3'5' $H 1 a$ and $F 3^{\prime} 5^{\prime} H 2 a$ reached maximum levels at $99 \mathrm{DAB}$ in Norton, and were significantly higher in Norton than in Cabernet Sauvignon post-véraison. Transcripts of DFR increased to the highest levels at véraison in both varieties, and then declined sharply in Cabernet Sauvignon, but remained at the same levels throughout the ripening stages in Norton. Transcripts of $L D O X$ were very low in Cabernet
Sauvignon, but in Norton they increased to a peak at 85 $\mathrm{DAB}$, declined at $99 \mathrm{DAB}$, and then bounced back to the same levels at $127 \mathrm{DAB}$ as at $85 \mathrm{DAB}$. UFGT transcript levels reached a maximum at $99 \mathrm{DAB}$, and also were significantly higher in Norton than in Cabernet Sauvignon (Figure 3).

Transcripts of $A N R$ attained peak levels at véraison, and declined gradually in Norton, but were significantly higher in Norton than in Cabernet Sauvignon post-véraison. Transcripts of $L A R 1$ were the most abundant at véraison, significantly higher in Cabernet Sauvignon than in Norton, and then declined to be barely detectable in the final two stages in Cabernet Sauvignon. In Norton, LAR1 transcript levels increased steadily after $85 \mathrm{DAB}$. On the other hand, LAR2 transcripts increased, and were also more abundant in Norton than in Cabernet Sauvignon post-véraison (Figure 3).

Taken together, transcripts of all eleven genes accumulated more abundantly in Norton after véraison, suggesting that the biosynthesis of flavonoid compounds remains highly activated in the skin of Norton berries post-véraison. 


\section{Expression pattern of GST and OMT}

In plants, GSTs consist of a large, complex gene family and play important roles in anthocyanin transport to or storage in the vacuole [54]. They conjugate the tripeptide glutathione to a variety of electrophilic compounds, thus limiting damaging effects of reactive oxygen species $[55,56]$. RNA-seq analysis showed that transcripts of 64 of the predicted 87 GSTs in grapevine were detected during berry development of the grape variety 'Corvina' [57]. However, the specific roles of the individual GSTs were not clear. Four GST isoforms were identified in cell suspension cultures of grapevine. Two of them were highly expressed and involved in anthocyanin accumulation or transport into the vacuole [58]. One grapevine GST (GSVIVT00023496001) gene was well-characterized [54], and was chosen for qPCR analysis of this gene family during berry skin development. We found that transcript levels of this GST gene reached a peak at $85 \mathrm{DAB}$ and declined slightly post-véraison, and were more abundant in Norton than in Cabernet Sauvignon berry skin (Figure 3). It is speculated that the difference in transcript levels of GST genes between the two varieties may lead to accumulation of more anthocyanins in the vacuoles of Norton berry skin cells than in those of Cabernet Sauvignon.

The methylation of phenolic compounds, as catalyzed by O-methyltransferases (OMTs), is an important step in flavonoid metabolism [59]. For example, caffeoyl CoA and caffeic acid OMTs are able to methylate lignin precursors $[60,61]$. On the basis of substrate specificity and function in stabilizing phenolic products, plant OMTs have been classified into various categories. Increasing evidence suggests that the expression of OMT genes is correlated with the accumulation of methylated anthocyanins in grapevines [62-64]. The qPCR results show that one OMT (GSVIVT00002831001) of grapevine was highly induced post-véraison when anthocyanins accumulated in both Cabernet Sauvignon and Norton. Transcript levels of this grapevine OMT were the highest at véraison, significantly higher in Cabernet Sauvignon than in Norton, and then declined gradually towards harvest (Figure 3). It is yet to be determined if this difference at transcript levels of this particular OMT could result in the production of different types of anthocyanin derivatives.

\section{Expression patterns of MYB transcription factors are unique in each variety}

To investigate transcriptional regulation of the flavonoid pathway during berry skin development, we analyzed the transcript levels of six genes encoding MYB transcription factors (MYBA1, MYBA2, MYBPA1, MYBPA2, $M Y B 5 A$ and $M Y B 5 B$ ) by qPCR (Additional File 7). All transcription factor genes assayed were expressed at some stages of berry skin development, but the expression patterns of some of them were distinct between the two varieties (Figure 4).

Expression profiles of $M Y B A 1$ and $M Y B A 2$ are very similar between the two varieties. MYBA1 transcripts reached peak levels at $85 \mathrm{DAB}$ after véraison in Norton and then declined and remained low. Similarly, the transcripts of $M Y B A 1$ reached the highest level at $59 \mathrm{DAB}$ (véraison) and decreased gradually post-véraison in Cabernet Sauvignon. MYBA2 transcripts also reached the highest level at $59 \mathrm{DAB}$, and then decreased until 112 DAB in Cabernet Sauvignon. In contrast, in Norton $M Y B A 2$ transcripts reached the highest level at $99 \mathrm{DAB}$.

The transcript profiles of $M Y B 5 A$ and $M Y B 5 B$ were similar during all of berry skin development, with high levels at véraison in both varieties. MYB5A transcript levels are slightly higher in Norton than in Cabernet Sauvignon while transcript levels of $M Y B 5 B$ are higher at all developmental stages in Cabernet Sauvignon than in Norton. The transcripts of MYBPA1 in Norton increased sharply from 66 to $71 \mathrm{DAB}$ (véraison), reached the highest level at $85 \mathrm{DAB}$, and then declined to a barely detectable level. The transcript levels of MYBPA1 in Cabernet Sauvignon, on the other hand, remained low throughout berry development. In contrast, MYBPA2 transcripts reached maximum levels at $71 \mathrm{DAB}$ in Cabernet Sauvignon, while they remained steadily low in Norton throughout berry development. The results suggest that MYBPA1 may play a more prominent role in Norton than in Cabernet Sauvignon whereas MYBPA2 in Cabernet Sauvignon than in Norton in the regulation of PA biosynthesis. The varietyspecific regulation of MYBPAs warrants further functional analysis of their regulatory elements.

\section{Proanthocyanidin and anthocyanin profiles in berry skin of Norton and Cabernet Sauvignon}

To match gene expression patterns with flavonoid profiles, we analyzed the accumulation of the flavan-3-ols catechin, epicatechin, epigallocatechin (EGC), and epicatechin gallate (ECG) in berry skin across seven developmental stages (Figure 5). Norton and Cabernet Sauvignon have comparative levels of catechin at 17 DAB. In Cabernet Sauvignon, catechin levels remained high until just after véraison, whereas in Norton, catechin dropped to the lowest levels at $71 \mathrm{DAB}$ (véraison) and then rose until 127 DAB. Epicatechin was not detected in either variety until véraison, but was detectable in Norton at 85 and 99 DAB as well as in Cabernet Sauvignon post-véraison. EGC levels remained steady in Cabernet Sauvignon throughout berry development, but increased steadily in Norton until 127 DAB. ECG was detected only in Cabernet Sauvignon (data not shown).

We analyzed the accumulation profiles of five anthocyanin derivatives (cyanidin-, peonidin-, delphinidin-, petunidin- and malvidin-monoglucoside/diglucoside) at 

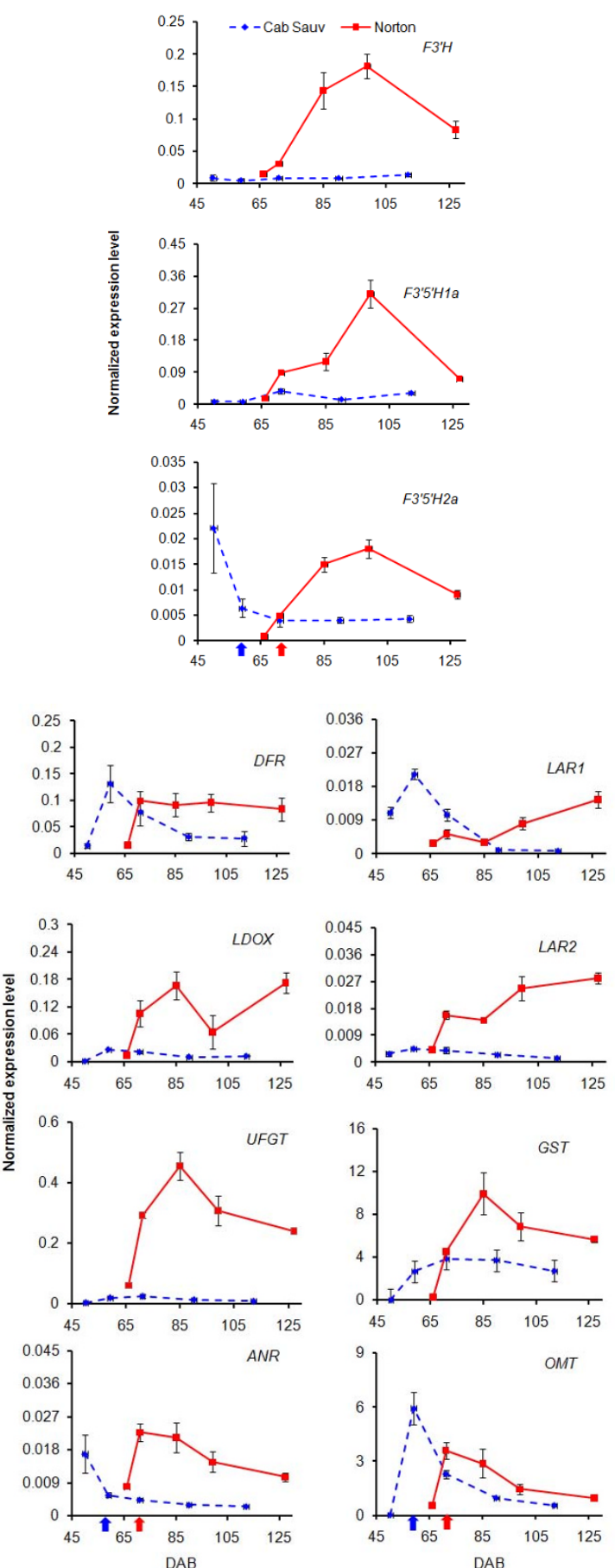

\begin{tabular}{|l|c|c|c|c|c|}
\hline & stage 34 & $\begin{array}{c}\text { stage 35 } \\
\text { (véraison) }\end{array}$ & stage 36 & stage 37 & stage 38 \\
\hline Cab. Sauv. & 49 & 59 & 71 & 90 & 112 DAB \\
\hline Norton & 66 & 71 & 85 & 99 & 127 DAB \\
\hline
\end{tabular}

Figure 3 Quantitative real-time (qPCR) assay of transcript abundance of the structural genes $F 3^{\prime} H, F 3^{\prime} 5^{\prime} H 1 a, F 3^{\prime} 5^{\prime} H 2 a, D F R, L D O X, U F G T$, ANR, LAR1, LAR2, GST and OMT in the flavonoid biosynthesis pathway during Vitis vinifera 'Cabernet Sauvignon' (blue dashed line) and V. aestivalis 'Norton' (red solid line) berry skin development. Cabernet Sauvignon berry skin were collected at 49, 59 (véraison, blue arrow), 71, 90 and 112 days after bloom (DAB), and Norton berry skin at 66, 71 (véraison, red arrow), 85, 99 and 127 DAB. Transcript abundance of each gene was normalized by the level of an actin gene. Bars indicate standard error of three biological replicates at each sampling time-point. 


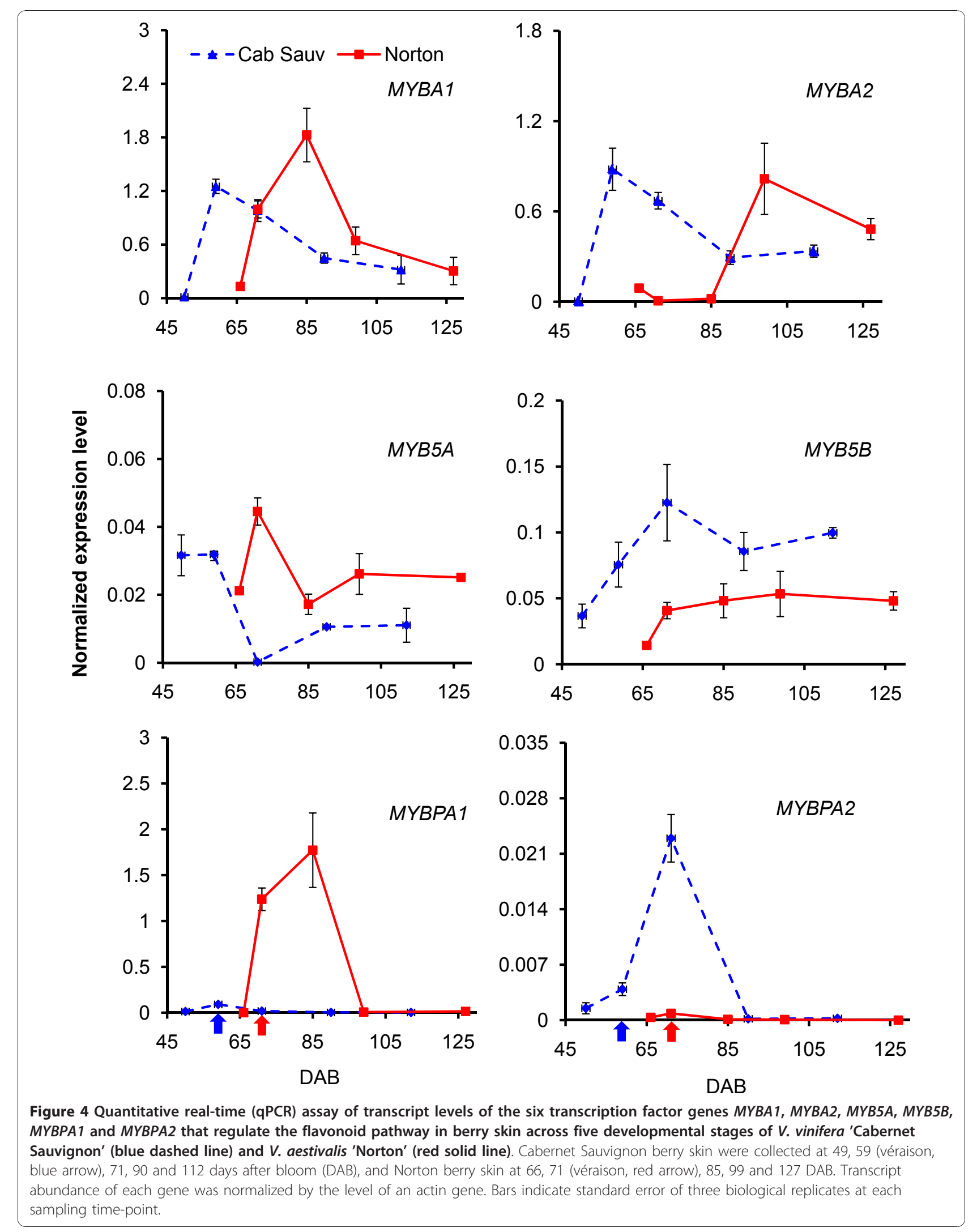



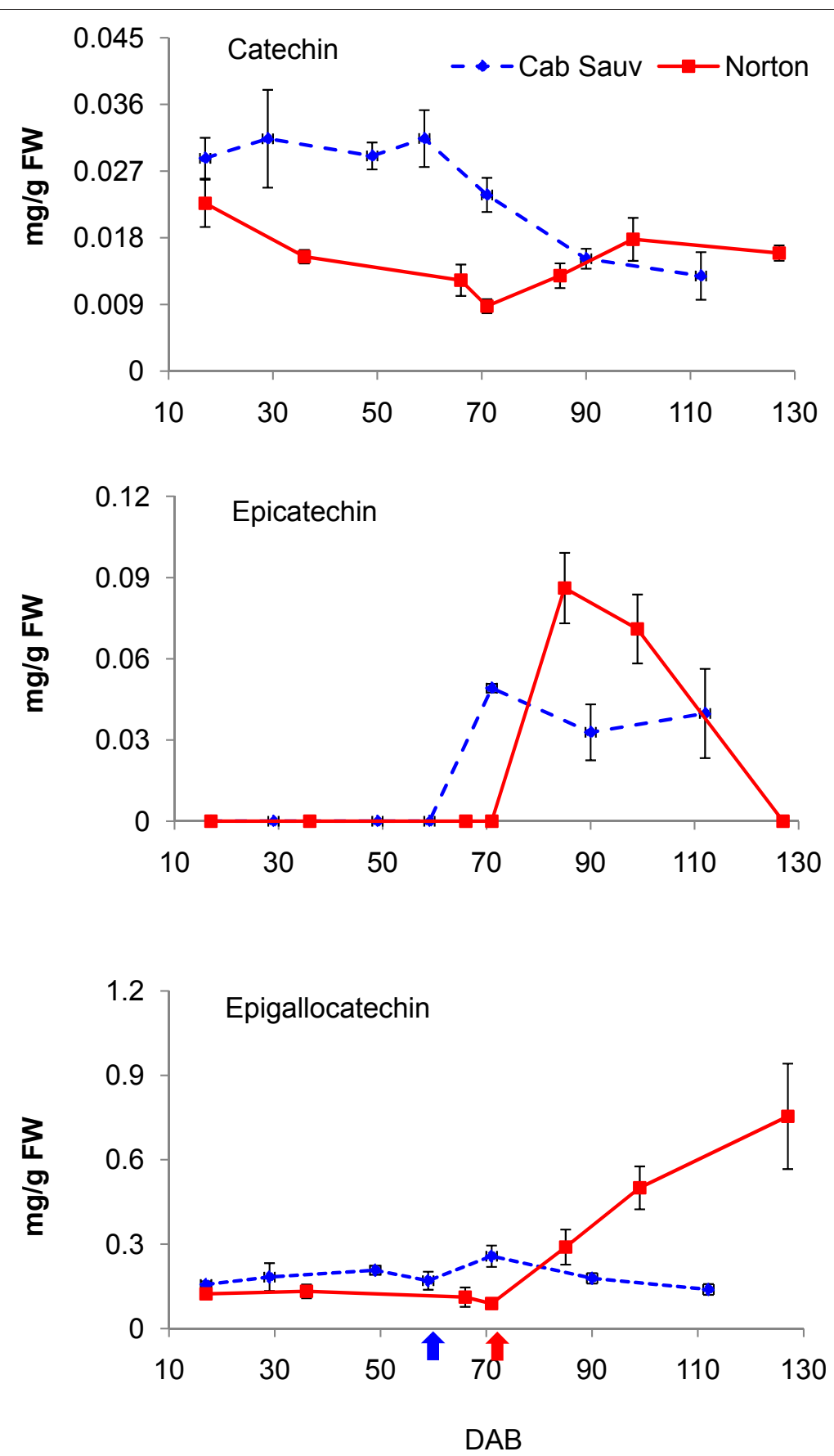

Figure 5 Accumulation kinetics of the proanthocyanidins catechin, epicatechin, and epigallocatechin during V. vinifera 'Cabernet Sauvignon' (blue dashed line) and V. aestivalis 'Norton' (red solid line) berry skin development. Cabernet Sauvignon berry skin were collected at 49, 59 (véraison, blue arrow), 71, 90 and 112 days after bloom (DAB), and Norton berry skin at 66, 71 (véraison, red arrow), 85, 99 and $127 \mathrm{DAB}$. Bars indicate standard error of three biological replicates per sample.

four post-véraison stages of berry skin for both varieties by high performance liquid chromatography (HPLC) (Figure 6). Accumulation patterns of the five anthocyanins in Cabernet Sauvignon berry skin in the present study are remarkably similar to the previous observations in Cabernet Sauvignon under different climate and environmental conditions [65]. The accumulation of the five anthocyanins begins at véraison, and leads to much higher levels in Norton than in Cabernet Sauvignon at harvest (Figure 6).

In agreement with previous results that diglucoside derivatives of anthocyanins are found in Vitis species of 

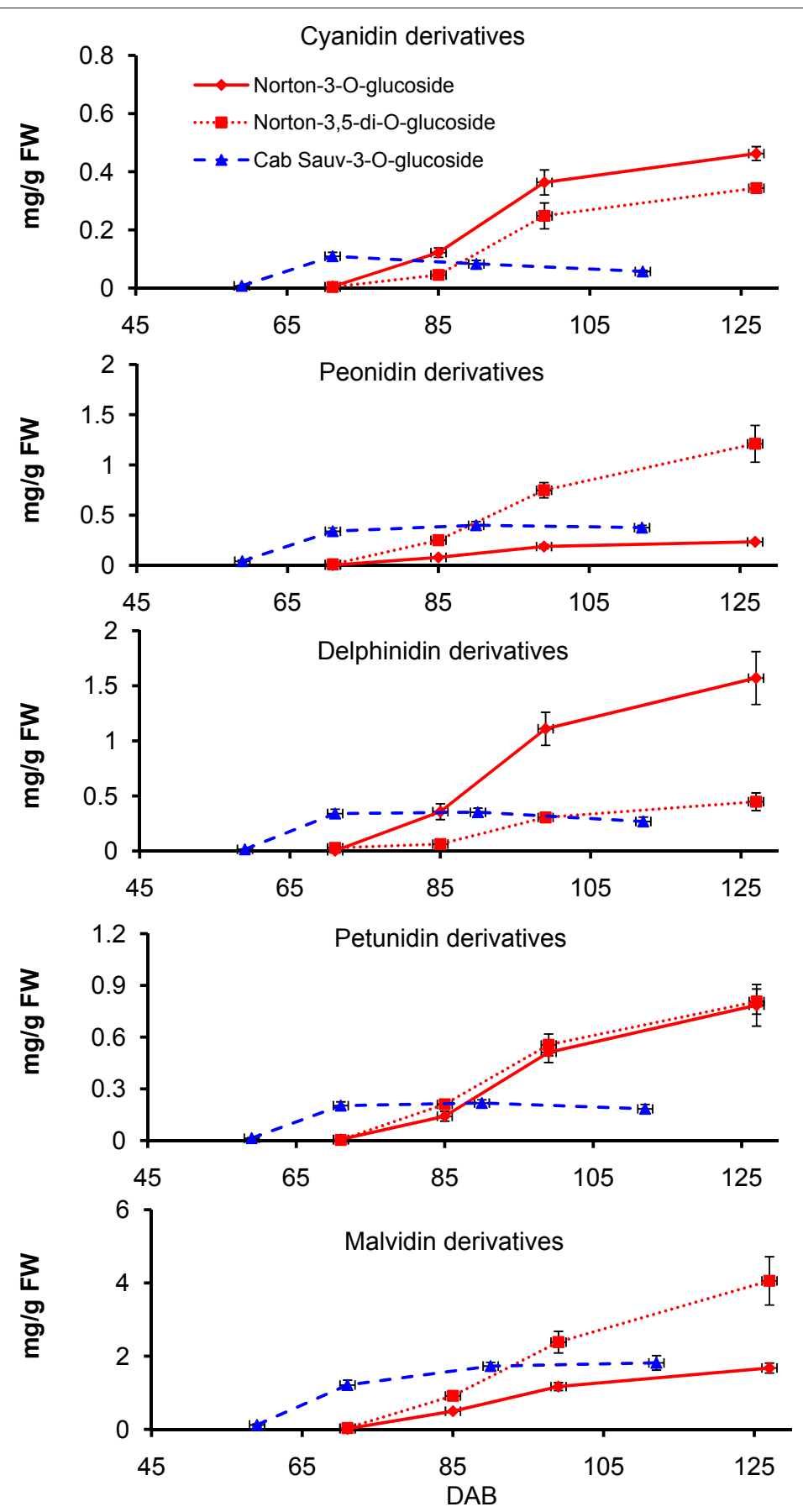

Figure 6 Accumulation kinetics of the anthocyanidin derivatives cyanidin, peonidin, delphinidin, petunidin and malvidin glucosides during V. vinifera 'Cabernet Sauvignon' (blue dashed line) and V. aestivalis 'Norton' (red solid line) berry skin development. Cabernet Sauvignon berry skin were collected at 49, 59 (véraison), 71, 90 and 112 days after bloom (DAB), and Norton berry skin at 66, 71 (véraison), 85, 99 and 127 DAB. Bars indicate standard error of three biological replicates per sample.

North American origin [66], anthocyanin-diglucosides are highly abundant and contribute a major portion to the total anthocyanin content in Norton berry skin (Figure 6). Interestingly, the amounts of monoglucoside derivatives of malvidin and peonidin are not significantly different between Norton at 127 DAB, and Cabernet
Sauvignon at 112 DAB. Diglucoside derivatives of peonidin and malvidin accumulated to significantly higher levels than their respective monoglucoside derivatives in Norton (Figure 6). Malvidin-diglucoside is the major anthocyanin in Norton while malvidin-monoglucoside contributes primarily to anthocyanin in Cabernet 
Sauvignon. The five anthocyanin derivatives reached their highest levels in Cabernet Sauvignon after véraison and remained steady until $112 \mathrm{DAB}$; whereas in Norton they continued to increase steadily until harvest at 127 DAB.

\section{Norton accumulates a broader spectrum of anthocyanins than Cabernet Sauvignon}

The differences detected in the accumulation of cyanidin-, peonidin-, delphinidin-, petunidin- and malvidin derivatives prompted us to compare anthocyanin profiles of ripe
Norton and Cabernet Sauvignon berry skin in detail. We used liquid chromatography-tandem mass spectrometry (LC-TIS/MS/MS) to identify the anthocyanin compounds. Thirty five different anthocyanins were identified in the two grape varieties (Table 3 and Figure 7). Eight of the 35 compounds were common to both varieties; sixteen of them were detected only in Norton. Norton-specific compounds include those previously described 3'-5' diglucoside derivatives as well as a number of sophoriside-glucosides and p-coumaryl-glucosides. Rutinoside derivatives appear to be

Table 3 Anthocyanins detected in the berry skin of ripe Norton and Cabernet Sauvignon grapes

\begin{tabular}{|c|c|c|c|}
\hline \multirow[t]{2}{*}{ Anthocyanins } & \multicolumn{2}{|c|}{ Compound ID ${ }^{A}$} & \multirow[t]{2}{*}{ Molecular ion: Product ion } \\
\hline & Norton & Cabernet Sauvignon & \\
\hline \multicolumn{4}{|l|}{ Compound detected in both varieties } \\
\hline Delphinidin 3-glucoside & 3 & 3 & 465: 303 \\
\hline Cyanidin 3-glucoside $^{B}$ & & 5 & 449 \\
\hline Petunidin 3-glucoside & 7 & 7 & 479: 317 \\
\hline Peonidin 3-glucoside & 9 & 9 & 463: 301 \\
\hline Malvidin 3-glucoside & 10 & 10 & 493: 331 \\
\hline Petunidin 3-(6"-acetylglucoside) & 17 & 17 & 521: 317 \\
\hline new pigment $\mathrm{B}$ & 33 & 33 & 677 \\
\hline Peonidin 3-O-cis-p-coumarylglucoside & 34 & 34 & 609 \\
\hline Malvidin 3-O-trans-p-coumarylglucoside & 35 & 35 & 639 \\
\hline \multicolumn{4}{|l|}{ Compound detected only in Norton } \\
\hline Delphinidin 3,5-diglucoside & 1 & & $627: 465,303$ \\
\hline Cyanidin 3,5-diglucoside & 2 & & 611: 449, 287 \\
\hline Peonidin 3,5-diglucoside & 4 & & 625: 463, 301 \\
\hline Malvidin 3,5-diglucoside & 6 & & 655: 493, 331 \\
\hline Delphinidin 3-arabinoside & 8 & & 435: 303 \\
\hline Malvidin 3-(6"-acetylglucoside)-5-glucoside & 11 & & 697: 535, 493, 331 \\
\hline Cyanidin 3-(acetylglucoside) & 14 & & 491: 287 \\
\hline Delphinidin-3-(6-O-p-coumarylglucoside)-5-glucoside & 16 & & 773: 611, 465, 303 \\
\hline Malvidin 3-sophoroside-5-glucoside & 19 & & 817: 655, 493, 331 \\
\hline Petunidin 3-(6"-p-coumarylglucoside)-5-glucoside & 21 & & 787: 625, 479, 317 \\
\hline Petunidin 3-sophoroside & 22 & & 641 \\
\hline Malvidin 3-(6"-acetylglucoside) & 23 & & 535: 331 \\
\hline Delphinidin 3-O-p-coumarylglucoside & 25 & & 611: 303 \\
\hline Malvidin 3-(6-O-p-coumarylglucoside)-5-glucoside & 26 & & 801: 639, 493, 331 \\
\hline Cyanidin 3-O-p-coumarylglucoside & 28 & & 595: 287 \\
\hline Petunidin 3-O-trans-p-coumarylglucoside & 31 & & 625: 317 \\
\hline \multicolumn{4}{|l|}{ Compound detected only in Cabernet Sauvignon } \\
\hline Delphinidin 3-(6"-acetylglucoside) & & 12 & 507: 303 \\
\hline Petunidin 3,7-di-glucoside & & 13 & 641 \\
\hline Delphinidin 3-O-beta-D-glucopyranoside & & 15 & 465 \\
\hline New pigment $A$ & & 18 & 573: 369 \\
\hline Peonidin 3-(6"-acetylglucoside) & & 20 & 505: 301 \\
\hline Cyanidin 3-(3"-malonylglucoside) & & 24 & 535 \\
\hline Petunidin 3-rutinoside & & 27 & 625: 301,317 \\
\hline Malvidin 3-gentiobiside & & 29 & 655: 331 \\
\hline Peonidin 3-rutinoside & & 30 & 609: 301 \\
\hline Malvidin 3-rutinoside & & 32 & 639: 331 \\
\hline
\end{tabular}

A The compound ID corresponds to the labels of the liquid chromatography peaks in Figure $7 .{ }^{\mathrm{B}}$ compound cyanidin 3-glucoside was detected in both varieties by HPLC with Agilent instrument as shown in Figure 6. 


\section{A. Cabernet Sauvignon}

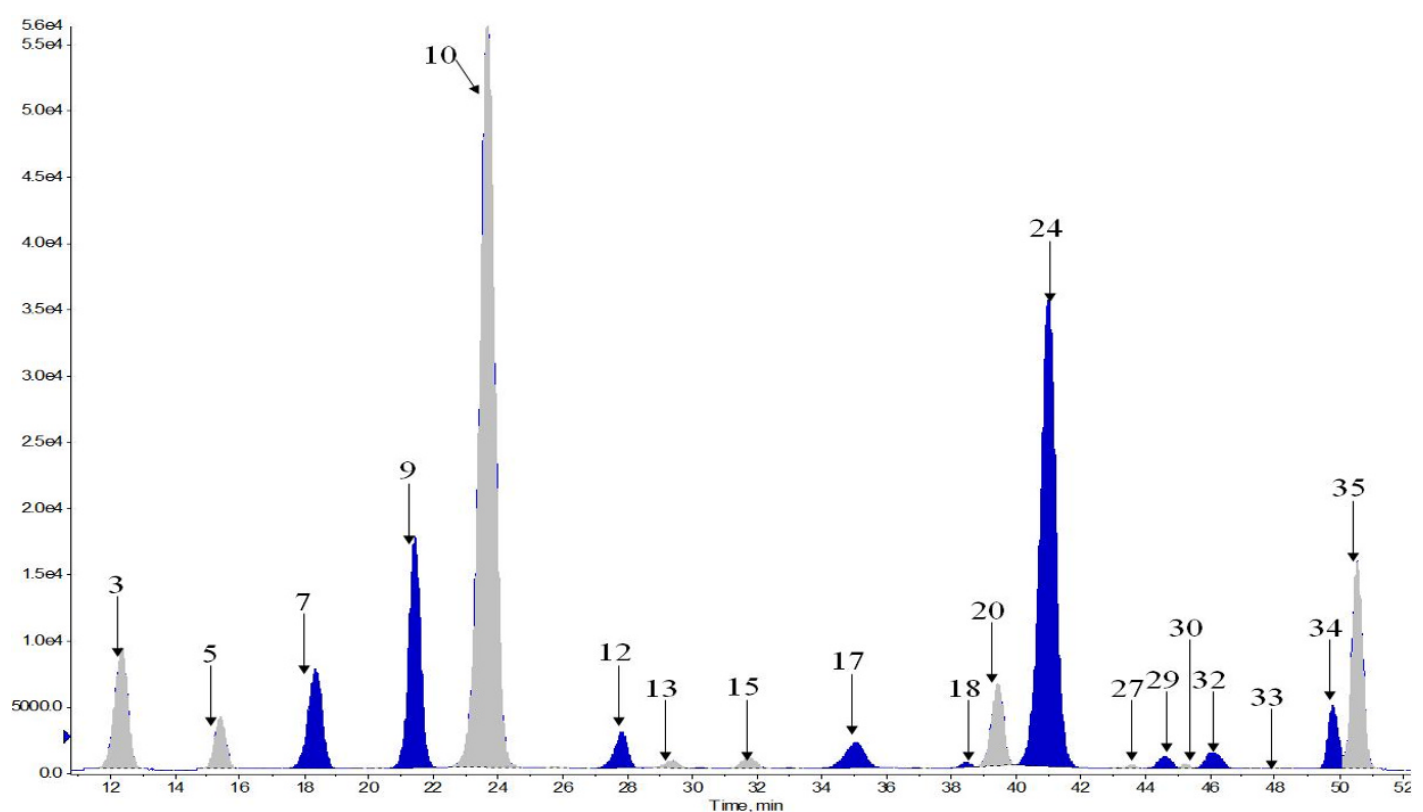

\section{B. Norton}

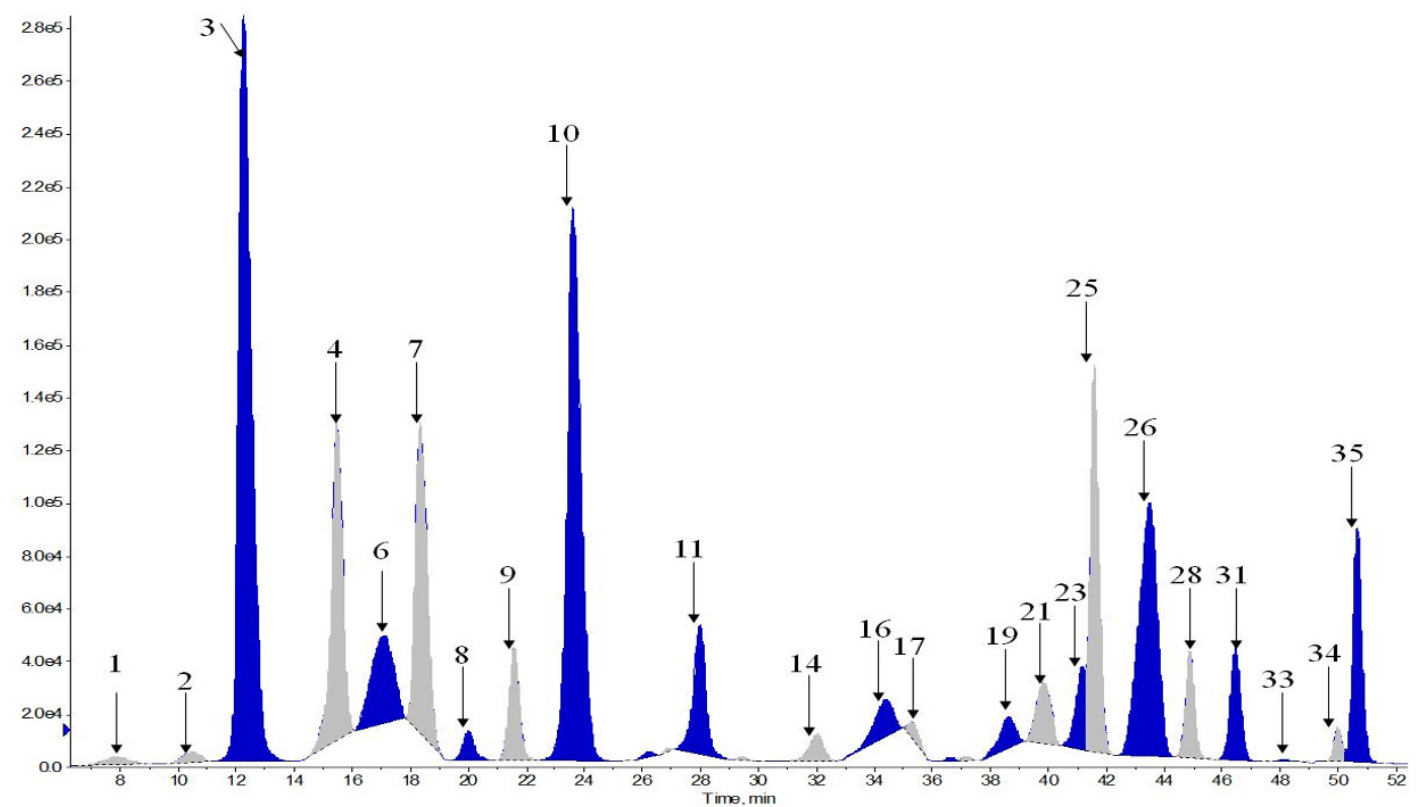

Figure 7 HPLC chromatograms of anthocyanin compounds in the berry skin of $V$. vinifera 'Cabernet Sauvignon' (A) and V. aestivalis 'Norton' (B) at harvest ripe (stage 38). More anthocyanin compounds were found in Norton berry skin than in Cabernet Sauvignon. The identified compounds from each profile are listed in Table 3. The HPLC conditions are described in Materials and Methods.

unique to Cabernet Sauvignon. Cabernet Sauvignon had a single diglucoside anthocyanin, namely petunidin $3^{\prime}, 7^{\prime}$ diglucoside (Table 3).

At $127 \mathrm{DAB}$, anthocyanin diglucosides contribute 59\% of the total anthocyanins in Norton berry skin. The major anthocyanins are malvidin derivatives that contribute $49 \%$ (5.73 mg/g FW) to total anthocyanins, followed by delphinidin (17\%), petunidin (11\%), peonidin (12\%), and cyanidin (7\%). In Cabernet Sauvignon, the main anthocyanin component is malvidin-3'-glucoside, which contributes 
$67 \%(1.82 \mathrm{mg} / \mathrm{g} \mathrm{FW})$ to the total anthocyanin amount at $112 \mathrm{DAB}$, followed by peonidin (14\%), delphinidin (10\%), petunidin $(6.8 \%)$ and cyanidin $(2 \%)$. Overall, in harvestripe berries, the total anthocyanin content in Norton berry skin (11.59 $\mathrm{mg} / \mathrm{g} \mathrm{FW})$ is considerably higher than in Cabernet Sauvignon berry skin $(2.70 \mathrm{mg} / \mathrm{g}$ FW).

\section{Expression profiles of key genes and accumulation of} anthocyanins and PAs display a good correlation in Norton berry skin

A concise summary of coordinated transcription of key genes and biosynthesis of anthocyanins and PAs in the developing berry skin is presented in Figure 8.
Transcript levels of F3' $H$ and F3'5'H1a/2a peaked at 99 $\mathrm{DAB}$ and were higher in Norton than in Cabernet Sauvignon (Figure 3 ). We speculate that more flavonoid precursors (dihydroflavonols) are produced that are converted to anthocyanins and PAs in Norton than in Cabernet Sauvignon. This speculation is supported by the patterns and levels of accumulation of anthocyanins and PAs during berry development of the two varieties (Figure 5 and 6). One DFR gene (GSVIVT00014584001) displayed enhanced expression at the onset of véraison and remained at steady levels in Norton berry skin postvéraison, as measured by both qPCR (Figure 4) and microarray analyses (cluster 1, Figure 1 and Table 2).

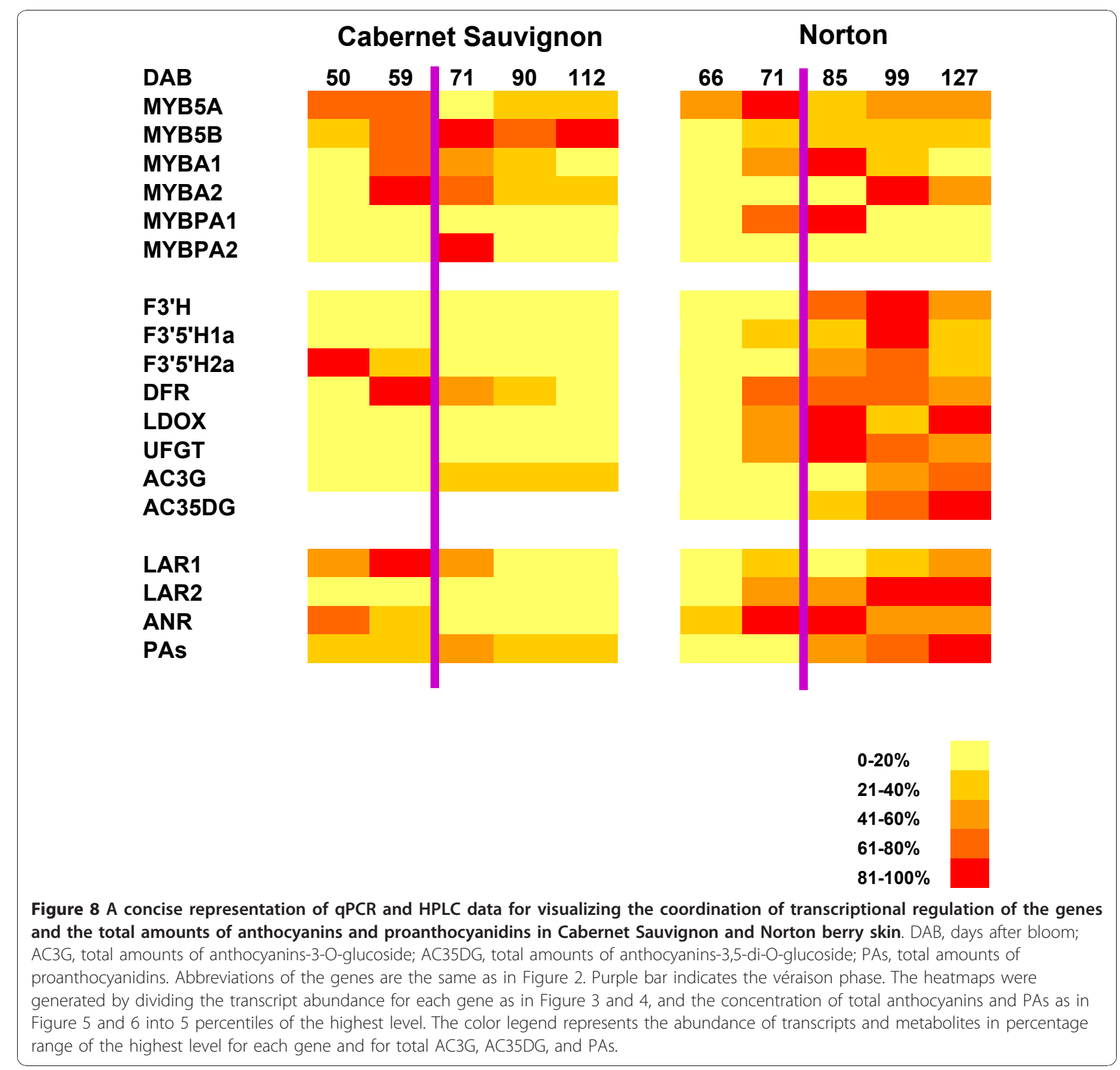


The constantly high mRNA levels of this DFR gene likely result in consistent production of leucoanthocyanidins that are substrates for LAR. Transcripts of LAR1 and LAR2 increased gradually after véraison (Figure 4), concurrently with catechin accumulation (Figure 5).

LDOX catalyzes the last two steps of anthocyanin synthesis (Figure 2B). The transcriptional profile of one LDOX gene (GSVIVT00001063001) showed increasing levels until $85 \mathrm{DAB}$, declining at $99 \mathrm{DAB}$, and increasing to the final stage in Norton berry skin, as observed in both microarray (cluster 13, Figure 1 and Table 2) and qPCR analyses (Figure 3). Transcripts of $L D O X$ are more abundant in Norton than in Cabernet Sauvignon throughout the ripening phase (Figure 3). The highest transcript levels of one ANR gene (GSVIVT00005344001) at the onset of véraison declined gradually during ripening (Figure 1, cluster 10 and Figure 3), which is in agreement with the pattern of epicatechin accumulation (Figure 5).

UFGT catalyzes the last step in the anthocyanin biosynthesis pathway (Figure 2B). MYBA1 and MYBA2 regulate the transcription of UFGT $[21,67,68]$. Transcript levels of MYBA1/A2 peaked at véraison (59 DAB) in Cabernet Sauvignon, and post véraison at 85 and 99 DAB in Norton. Correspondingly, transcripts of one UFGT gene (GSVIVT00014047001) reached maximum levels at $85 \mathrm{DAB}$ in Norton, but were found to be at significantly lower levels in Cabernet Sauvignon. The synchronized expression patterns of MYBA1/A2 and UFGT in both varieties suggest a close correlation between the transcription factors and their target genes. The higher transcript levels of UFGT in Norton than in Cabernet Sauvignon post-véraison (Figure 3) correlate remarkably well with the higher content of total anthocyanins in Norton berry skin at harvest (Figure 6).

\section{Conclusions}

In summary, developmentally regulated resistance of Norton ripening berry to pathogens likely is a result of the steady increase of transcript abundance of $R$ genes, $P R-1$, stilbene synthase genes, and genes of the phenylpropanoid pathway along the berry skin development. The expression patterns of six MYB transcription factor genes and their target structural genes in the anthocyanin and PA biosynthesis pathways correlate highly with the accumulation patterns of three PA compounds and five classes of anthocyanins. MYBPA1 and MYB5A may play more significant roles in the regulation of the flavonoid biosynthesis pathway in Norton than in Cabernet Sauvignon, whereas MYBPA2 and MYB5B appear to be more important in Cabernet Sauvignon than in Norton. The concomitant modulation of anthocyanin biosynthesis at the transcriptional level leads to more abundant production of anthocyanins in Norton berry skin in comparison with Cabernet Sauvignon berry skin.

\section{Methods}

\section{Collection of berry skin}

Berries from $V$. vinifera 'Cabernet Sauvignon' and $V$. aestivalis 'Norton' were collected at six developmental stages during the 2008 growing season from vines grown in a vineyard in the Missouri State Fruit Experiment Station, Mountain Grove, Missouri, USA, according to the phenological developmental stages defined by Coombe [69]. The berries were sampled at the following stages: 31 (pea-sized), 33 (still hard), 34 (softening), 35 (véraison), followed by 36,37 and 38 (harvest ripe). Berry skin was separated from pulp, and pulp tissues were further removed by rubbing the internal side of the skin against filter paper. The cleaned skin tissues were immediately frozen in liquid nitrogen and stored at $-80^{\circ} \mathrm{C}$.

\section{RNA extraction and cDNA synthesis}

Total RNA was extracted from the skin tissue according to the procedure of Reid et al. [70], using a CTABspermidine extraction buffer. Total RNA was treated with 1 unit of DNase I (Ambion, Austin, Texas, USA) for 30 minutes at $37^{\circ} \mathrm{C}$ and purified using RNeasy MinElute Cleanup kit (Qiagen, Valencia, California, USA). RNA quantity and quality were assessed by Agilent 2100 Bioanalyzer (Agilent Technologies, Santa Clara, California, USA). For cDNA synthesis, two $\mu \mathrm{g}$ of total RNA was reverse transcribed with oligo-dT in a $20 \mu \mathrm{l}$ reaction mixture using the MultiScribe reverse transcriptase (Applied Biosystems, Branchburg, New Jersey, USA) according to the manufacturer's instructions.

\section{Microarray hybridization and data processing}

Array hybridization was performed at the DNA Core Facility, University of Missouri (Columbia, Missouri). A total of $0.5 \mu \mathrm{g}$ of total RNA was used to make the biotin-labeled antisense RNA (aRNA) target using the MessageAmp $^{\text {ma }}$ Premier RNA amplification kit (Ambion, Austin, Texas) following the manufacturer's protocol. Briefly, total RNA was reverse transcribed to first strand cDNA with an oligo(dT) primer bearing a 5'-T7 promoter using ArrayScript reverse transcriptase. First strand cDNA then underwent second-strand synthesis to convert it into double stranded cDNA as a template for in vitro transcription. The biotin-labeled aRNA was synthesized using T7 RNA transcriptase with biotin-NTP mix. After purification, the aRNA was fragmented in $1 \times$ fragmentation buffer at $94^{\circ} \mathrm{C}$ for $35 \mathrm{~min}$. One hundred and thirty $\mu \mathrm{L}$ of hybridization solution containing $50 \mathrm{ng} / \mu \mathrm{l}$ of fragmented aRNA was hybridized to the Affymetrix GRAPEGEN GeneChip (Affymetrix, Santa Clara, California) at $45^{\circ} \mathrm{C}$ for $20 \mathrm{hrs}$. After hybridization, the chips were washed and stained with R-phycoerythrin-streptavidin in an Affymetrix fluidics station 450 using fluidics protocol Midi_euk2v3-450. The 
image data were acquired by Affymetrix GeneChip scanner 3000 and Affymetrix GCOS software.

\section{Annotation of probe sets and clustering}

The Affymetrix microarray (GRAPEGEN GeneChip) used in this analysis included probe sets for 23,096 unigenes [30]. The intensity data of all genes on the microarray were analyzed by ANOVA with the BenjaminiHochberg False Discovery Rate Multiple Test Correction method and applying a $p$-value of 0.001 . The resulting data set was further reduced by applying a cut-off fold change of 2 or greater, which led to a final set of 3,352 significantly changed probe sets.

To annotate the putative function of the 3,352 probe sets that exhibited significant expression changes during berry development, the FASTA sequences were BLATsearched against the $8 \times$ genomic sequences of $V$. vinifera PN40024 (http://www.genoscope.cns.fr/externe/GenomeBrowser/Vitis/) by using each FASTA sequence as query to acquire a Genoscope ID number. If no Genoscope ID was found for the query sequence, a Tentative Consensus (TC) ID was retrieved from VVGI5 database (http://compbio.dfci.harvard.edu/tgi/cgi-bin/tgi/gimain. $\mathrm{pl}$ ?gudb=grape). The latest annotations for all Genoscope IDs and relational Network IDs, InterPro domain IDs, Gene Ontology IDs, UniProtIDs, TCs and functions have been published (Table S1, [71]), and were used as the reference for functional category and annotation. The original annotations by the GeneChip manufacturing group were also cross-referenced for verification.

More than one sequence was annotated with the identical Genoscope or DFCI ID in 401 cases, which brought the total number of unigenes down to 2,760. All genes with multiple annotations and four sequences for which neither a Genoscope annotation nor a DFCI match were found were removed from the data set, resulting in 2,359 unigenes.

The expression profiles of the 2,359 unigenes were clustered using the $k$-means method with Pearson's correlation as distance. They were grouped into 20 clusters after evaluation of the Figure of Merit (FOM) graph in the Multiple Experiment Viewer version 4.4 software package.

\section{Quantitative real-time PCR (qPCR)}

Transcript levels in grape skin were measured by quantitative real-time PCR, using SYBR Green in the MX3005P system (Stratagene) following the manufacturer's manual. The reaction mixture $(20 \mu \mathrm{l}$, in triplicate) contained $0.5 \mu \mathrm{l}$ 1:10 diluted cDNA as a template and 20 pmole each of the forward and reverse primers specific to each gene. The primers were designed from the 3'-UTR region to avoid any unspecific amplification. Thermal cycling conditions were as follows: $95^{\circ} \mathrm{C}$ for
$10 \mathrm{~min}, 65$ cycles of $95^{\circ} \mathrm{C}$ for $15 \mathrm{sec}, 60^{\circ} \mathrm{C}$ for $30 \mathrm{sec}$ and 1 cycle of $95^{\circ} \mathrm{C}$ for $1 \mathrm{~min}, 60^{\circ} \mathrm{C}$ for $30 \mathrm{sec}$ and $95^{\circ} \mathrm{C}$ for $30 \mathrm{sec}$. The annealing temperature $\left(60^{\circ} \mathrm{C}\right)$ was determined computationally when designing the primer. The melt curves for the products of these assays produced a single peak, indicating that a single gene had been amplified. The specificity of each primer pair was also checked by gel electrophoresis and by sequencing the PCR products and comparing them with the sequence of the target gene. PCR efficiency $(E)$ was calculated from the exponential phase of each individual amplification plot and the equation $(1+E)=10^{\text {slope }}$ based on a previous method [72]. Expression levels of genes of interest (GOI) were normalized to that of ACTIN by dividing the $\mathrm{C}_{\mathrm{T}}$ value of GOI by the $\mathrm{C}_{\mathrm{T}}$ value of $A C T I N$. Gene expression was expressed as mean and standard error calculated based on three biological replicates.

\section{Reverse phase HPLC analysis of anthocyanins and proanthocyanidins}

For anthocyanin extraction, frozen berry skin tissue was ground in liquid nitrogen, and $500 \mathrm{mg}$ of the ground tissue was extracted with $5 \mathrm{~mL}$ acidified methanol (60\% $(\mathrm{V} / \mathrm{V})$ methanol containing $0.1 \%(\mathrm{w} / \mathrm{V})$ ascorbic acid) for 24 hours on a shaker in the dark at room temperature. The extracts were centrifuged twice at $16,100 \mathrm{~g}$ for 10 minutes. The final supernatants were kept in the dark and refrigerated until analysis; two samples were prepared from each biological replicate.

For proanthocyanidin extraction, frozen seeds or frozen berry skin were ground in liquid nitrogen, and $500 \mathrm{mg}$ of ground tissue was used for extraction in $5 \mathrm{ml}$ extraction buffer $(70 \%[\mathrm{~V} / \mathrm{V}]$ acetone containing $0.1 \%$ [w/V] ascorbic acid) for $24 \mathrm{hr}$ at room temperature on a rotating shaker in darkness. The water phase was separated from the acetone phase by adding sodium chloride to saturation. After removal of the acetone phase, the water phase was extracted with additional sodium chloride-saturated $100 \%$ acetone, and the resulting acetone phase was combined with the first acetone phase. The samples were dried under a stream of nitrogen, the pellet re-dissolved in $750 \mu \mathrm{L}$ of $60 \%$ methanol acidified with $0.1 \%$ ascorbic acid, centrifuged at $16,100 \mathrm{~g}$ for 10 minutes, and the final supernatant kept in darkness and under refrigeration until analysis; two samples were prepared from each biological replicate.

Anthocyanin and proanthocyanidin content and composition were determined by reverse-phase HPLC using an HP1100 series (Agilent) Chemstation, with a Zorbax Eclipse XDB-C18 (80 Angstrom, $4.6 \times 150 \mathrm{~mm}$, particle size $3 \mu \mathrm{m}$ ) column with a guard column. The binary solvent system of solvent A (acetonitrile (HPLC grade, EMD Chemicals, USA) and Solvent B (2\% phosphoric acid [(HPLC grade, Sigma Aldrich), V/V Millipore 
water] was used for both the anthocyanin and the proanthocyanidin analyses. The gradient used for anthocyanin separation was as follows: acetonitrile $6 \%$ for $3 \mathrm{~min}$; $8 \%$ for $24.50 \mathrm{~min}$; $10 \%$ for $22.50 \mathrm{~min}$; $18 \%$ for 23.50 min; $90 \%$ for $4.5 \mathrm{~min}$; and $8 \%$ for $7 \mathrm{~min}$; with a flow rate of $0.8 \mathrm{~mL} / \mathrm{min}$ for 36 minutes, then $0.6 \mathrm{~mL} / \mathrm{min}$ for 49 min. The gradient used for proanthocyanidin separation was as follows: acetonitrile $8 \%$ for $5 \mathrm{~min}, 12 \%$ for $12 \mathrm{~min}$, $20 \%$ for $10 \mathrm{~min}$, $25 \%$ for $6 \mathrm{~min}, 50 \%$ for $2 \mathrm{~min}, 80 \%$ for 7 $\mathrm{min}, 8 \%$ for $5 \mathrm{~min}$; with a flow rate of $0.5 \mathrm{~mL} / \mathrm{min}$. In each case, the column was maintained at $40^{\circ} \mathrm{C}$ and the diode array detector was used to record absorption at $280 \mathrm{~nm}$, $335 \mathrm{~nm}$ and $520 \mathrm{~nm}$. Malvidin-3-glucoside chloride, catechin hydrate, epicatechin, epicatechin gallate, epigallocatechin, epigallocatechin gallate and proanthocyanidin B2 (all HPLC grade, Sigma-Aldrich) were used to create standard absorption curves. All anthocyanins were expressed as malvidin glucoside equivalents based on the peak areas recorded at $520 \mathrm{~nm}$ with a molecular weight correction factor applied. The peak areas recorded at $280 \mathrm{~nm}$ in conjunction with the respective standard absorption curves were used to express the proanthocyanidins as $\mathrm{mg}$ per gram of fresh weight.

\section{LC-TIS/MS/MS analysis of anthocyanins}

Anthocyanins were extracted by following the protocol for extracting proanthocyanidins as described in the previous section. All samples were analyzed using a 4000 QTRAP LC-TIS-MS-MS system (Applied Biosystems, Forest City, CA) by monitoring the enhanced product ion (EPI) and multiple reaction monitoring (MRM) in the positive ionization mode. Separation of $(10 \mu \mathrm{L})$ samples was achieved by using a Gemini-NX C18 HPLC column (Phenomenex, $5 \mu \mathrm{m}, 150 \mathrm{~mm} \times 2 \mathrm{~mm}$ ) combined with a C18 guard column (Phenomenex, $4 \mathrm{~mm} \times 2 \mathrm{~mm}$ ). The mobile phase flow was set to $0.45 \mathrm{~mL} / \mathrm{min}$ with binary gradient elution, using solvent A (aqueous 5\% formic acid solution) and B (95\% $\mathrm{CH}_{3} \mathrm{CN}, 5 \%$ formic acid). The gradient was as follows: 0-3 $\mathrm{min}, 5 \% \mathrm{~B} ; 3-15 \mathrm{~min}, 5-9 \% \mathrm{~B} ; 15-27 \mathrm{~min}$, 9-13.5\% B; 27-32 min, $13.5 \%$ B, 32-42 min, $13.5-18.5 \%$ B; 42-44 min, $18.5 \%$ B; 44-51 min, $18.5-22.5 \%$ B; 51-55 min, 22.5-30\% B; 55-56 min, 30-40\% B; 56-60 min, 40-70\%; 60-60.1 min, 70-100\% B; 60.1-70 min, $100 \%$ B; 70.0-70.1 min, $100-5 \%$ B; $70.1-80$ min, $5 \%$ B. The elution of anthocyanins was monitored at $520 \mathrm{~nm}$. The following TIS source parameters were used: CUR $30 \mathrm{eV}$, CAD high, IS 5500, TEM $550^{\circ} \mathrm{C}$, DP $40 \mathrm{eV}, \mathrm{CE} 10 \mathrm{eV}$. The mass scan range was 50 to 1000 . For anthocyanin quantification, five anthocyanin standards (Chloride salt of delphinidin (Sigma, MO), cyanidin (Chromadex, CA), petunidin (Chromadex, CA), peonidin (Chromadex, CA) and malvidin (Chromadex, CA) were used to create a calibration curve for each anthocyanin. All calibration curves were linear, with $R^{2} \geq 0.998$.

\section{Additional material}

Additional file 1: Principal Component Analysis (PCA) of the eighteen set of microarray hybridization data. Six stages (Stage 33 to 38) are denoted by different colors. Filled rectangle, rectangle, and filled circle represent three biological replicates.

Additional file 2: Hierarchical cluster analyses of the eighteen sets of data for assessing the quality of the data.

Additional file 3: Pearson correlation coefficient analysis of the eighteen set of data in pair-wise.

Additional file 4: A list of 15,823 probe sets that exhibited significant variations along six stages (at $p$-value $\leq \mathbf{0 . 0 0 1}$ ). This list of probe sets was generated by conducting ANOVA on error-weighted intensity experiment definitions (EDS). Sequence description: Brief narrative description of gene annotation; Grand average: the average value of each probe set intensity across all factor levels in the ANOVA, and this average was computed after error-weighting; The Pooled Variance: the within mean square for each gene-analysis level item across all factor levels; Group p-value: the probability that the null hypothesisthat expression levels or differential expression ratio levels are not significantly different across factor levels-is not true. A low p-value indicates high confidence that the gene's expression level or ratio level is significantly different across the groups defined in the ANOVA.

Additional file 5: A list of 3,352 probe sets that exhibited significant variations along six stages (at $p$-value $\leq 0.001$ ) with a ratio of more than 2 . The legends of each column are the same as in Additional file 4. This list of probe sets was determined by conducting error-weighted ANOVA.

Additional file 6: Cluster analysis of the transcript abundance of the differentially expressed 2,359 unigenes across six developmental berry skin stages.

Additional file 7: GenBank accession number, Genoscope number, TC number, GeneChip ID number, primer sequences, expected size and sequences of amplified DNA fragments of the genes that were analyzed in the berry skin of Norton and Cabernet Sauvignon by the quantitative real-time PCR (qPCR). The qPCR-amplified DNA fragments were sequenced to verify the identity of each amplicon. Correlation coefficient analysis of the transcript levels between GPCR and microarray was also included.

\section{Abbreviations}

PAL: phenylalanine ammonia-lyase; $C 4 \mathrm{H}$ : cinnamate 4-hydroxylase; 4CL: 4-coumarate-CoA ligase; CAD: cinnamyl alcohol dehydrogenase; CCOAOMT: caffeoyl-COA 3'-O-methyltransferase; COMT: caffeic acid O-methyltransferase; CCR: cinnamoyl-CoA reductase; F5H: ferulate-5'-hydroxylase; STS: stilbene synthase; CHS: chalcone synthase; CHI: chalcone isomerase; UFGT: UDPglucose:flavonoid-3-O-glucosyltransferase; $\mathrm{F3H}$ : flavanone 3-hydroxylase; F3'H: flavonoid-3'-O-hydroxylase; F3'5'H: flavonoid-3',5'-hydroxylase; DFR: dihydroflavonol-4-reductase; LDOX: leucoanthocyanidin dioxygenase; ANR: anthocyanidin reductase; LAR: leucoanthocyanidin reductase; GST: glutathione S-transferase; OMT: O-methyltransferase; PA: proanthocyanidin.

\section{Acknowledgements}

This project was supported mainly by Missouri Life Science Research Board grant (No. 13234) to L.G. K., O.Y. and W. Q., and also by USDA-CSREES (200938901-19962) grant to L.G.K. and W.Q. as well as DOE (DE-SC0001295), NSF (MCB-0923779) and USDA (2010-65116-20514) grants to O.Y. We thank Patrick Hurban, formerly at Beckman Coulter Genomics, Morrisville, North Carolina, USA, for providing statistical analyses of the microarray data. We thank staff members at the DNA Core Facility, University of Missouri (Columbia, Missouri, USA) for performing array hybridizations and Daniel Ruzicka for RNA quantity/quality analysis. We are indebted to Walter Gassmann and Chin-Feng Hwang for reviewing the manuscript and Jennifer Howard for editing the manuscript.

The microarray data have been submitted to Gene Expression Omnibus under the access number GSE24561. 


\section{Author details}

${ }^{1}$ Center for Grapevine Biotechnology, William H. Darr School of Agriculture, Missouri State University, Mountain Grove, MO 65711, USA. ${ }^{2}$ The Donald Danforth Plant Science Center, St. Louis, MO 63132, USA. ${ }^{3}$ College of Food Sciences and Nutritional Engineering, China Agricultural University, Beijing 100083, PR China. ${ }^{4}$ Department of Plant and Soil Sciences, University of Kentucky, Lexington, KY 40546, USA.

\section{Authors' contributions}

MBA extracted total RNA, analyzed RNA quality, performed qPCR, made graphs and assisted in annotating genes, analyzing data and drafting manuscript. SH collected samples, performed chemical analysis of berries, clustering of microarray data and statistical analysis of qPCR results, conducted HPLC, and assisted in annotation of genes. SC established and optimized HPLC conditions for analyzing anthocyanins and PAs. YW and OY performed LC-TIS/MS/MS analysis. LGK conceived, designed and supervised the experiments, collected samples and contributed to manuscript writing. WQ conceived the comparative study between the two grape varieties; supervised QPCR assays, annotation and clustering of genes, and drafted and finalized the manuscript. All authors were involved in editing and revising the manuscript.

Received: 12 October 2010 Accepted: 10 January 2011

Published: 10 January 2011

\section{References}

1. Coombe B, McCarthy M: Dynamics of grape berry growth and physiology of ripening. Aust J Grape Wine Res 2000, 6:131-135.

2. Conde C, Silva P, Fontes N, Dias A, Tavares R, Sousa M, Agasse A, Delrot S, Geros H: Biochemical changes throughout grape berry development and fruit and wine quality. Food 2007, 1:1-22.

3. Davies C, Boss P, Robinson S: Treatment of grape berries, a nonclimacteric fruit with a synthetic auxin, retards ripening and alters the expression of developmentally regulated genes. Plant Physiol 1997, 115:1155-1161.

4. Symons G, Davies C, Shavrukov Y, Dry I, Reid J, Thomas M: Grapes on steroids. Brassinosteroids are involved in grape berry ripening. Plant Physiol 2006, 140:150-158.

5. Chervin C, El-Kereamy A, Roustan J, Latche A, Lamon J, Bouzayen M: Ethylene seems required for the berry development and ripening in grape, a non-climacteric fruit. Plant Sci 2004, 167:1301-1305.

6. Coombe B: Research on development and ripening of the grape berry. Am J Enol Vitic 1992, 43:101-110.

7. Deluc L, Grimplet J, Wheatley M, Tillett R, Quilici D, Osborne C, Schooley D, Schlauch K, Cushman J, Cramer G: Transcriptomic and metabolite analyses of Cabernet Sauvignon grape berry development. BMC Genomics 2007, 8(1):429.

8. Pilati S, Perazzolli M, Malossini A, Cestaro A, Dematte L, Fontana P, Dal Ri A, Viola R, Velasco R, Moser C: Genome-wide transcriptional analysis of grapevine berry ripening reveals a set of genes similarly modulated during three seasons and the occurrence of an oxidative burst at veraison. BMC Genomics 2007, 8(1):428.

9. Grimplet J, Deluc L, Tillett R, Wheatley M, Schlauch K, Cramer G, Cushman J: Tissue-specific mRNA expression profiling in grape berry tissues. BMC Genomics 2007, 8:187.

10. Dixon RA, Xie DY, Sharma SB: Proanthocyanidins: a final frontier in flavonoid research? New Phytol 2005, 165:9-28.

11. Adams DO: Phenolics and Ripening in Grape Berries. Am J Enol Vitic 2006, 57(3):249-256.

12. Ficke A, Gadoury DM, Seem RC, Dry IB: Effects of ontogenic resistance upon establishment and growth of Uncinula necator on grape berries. Phytopathology 2003, 93:556-563.

13. Ficke A, Gadoury DM, Seem RC, Godfrey D, Dry IB: Host barriers and responses to Uncinula necator in developing grape berries. Phytopathology 2004, 94:438-445.

14. Gadoury DM, Seem RC, Ficke A, Wilcox WF: Ontogenic resistance to powdery mildew in grape berries. Phytopathology 2003, 93:547-555.

15. Deytieux C, Geny L, Lapaillerie D, Claverol S, Bonneu M, Doneche B: Proteome analysis of grape skins during ripening. J Exp Bot 2007, 58:1851-1862.
16. Davies $C$, Robinson S: Differential screening indicates a dramatic change in mRNA profiles during grape berry ripening. Cloning and characterization of cDNAs encoding putative cell wall and stress response proteins. Plant Physiol 2000, 122:803-812.

17. Pozo Od, Lam E: Caspases and programmed cell death in the hypersensitive response of plants to pathogens. Current Biology 1998, 8:1129-1132.

18. Tattersall D, Van Heeswijck R, Hoj P: Identification and characterization of a fruit-specific, thaumatin-like protein that accumulates at very high levels in conjunction with the onset of sugar accumulation and berry softening in grapes. Plant Physiol 1997, 114(3):759-769.

19. Salzman RA, Tikhonova I, Bordelon BP, Hasegawa PM, Bressan RA: Coordinate accumulation of antifungal proteins and hexoses constitutes a developmentally controlled defense response during fruit ripening in grape. Plant Physiol 1998, 117:465-472.

20. Cos P, De Bruyne T, Hermans N, Apers S, Berghe DV, Vlietinck AJ: Proanthocyanidins in health care: current and new trends. Curr Med Chem 2004, 11:1345-1359.

21. Kobayashi S, Goto-Yamamoto N, Hirochika H: Retrotransposon-induced mutations in grape skin color. Science 2004, 304:982.

22. Bogs J, Jaffe FW, Takos AM, Walker AR, Robinson SP: The grapevine transcription factor VvMYBPA1 regulates proanthocyanidin synthesis during fruit development. Plant Physio 2007, 143:1347-1361.

23. Walker AR, Lee E, Bogs J, McDavid DAJ, Thomas MR, Robinson SP: White grapes arose through the mutation of two similar and adjacent regulatory genes. Plant J 2007, 49:772-785.

24. Deluc L, Barrieu F, Marchive C, Lauvergeat V, Decendit A, Richard T, Carde JP, Merillon JM, Hamdi S: Characterization of a grapevine R2R3MYB transcription factor that regulates the phenylpropanoid pathway. Plant Physiol 2006, 140(2):499-511.

25. Terrier N, Torregrosa L, Ageorges A, Vialet S, Verries C, Cheynier V, Romieu C: Ectopic expression of VvMybPA2 promotes proanthocyanidin biosynthesis in grapevine and suggests additional targets in the pathway. Plant Physio 2009, 149:1028-1041.

26. Fung RWM, Gonzalo M, Fekete $C$, Kovacs LG, He Y, Marsh E, Mclntyre LM, Schachtman DP, Qiu WP: Powdery mildew induces defense-oriented reprogramming of the transcriptome in a susceptible but not in a resistant grapevine. Plant Physio 2008, 146:236-249.

27. Hogan S, Zhang L, Li J, Zoecklein B, Zhou K: Antioxidant properties and bioactive components of Norton (Vitis aestivalis) and Cabernet Franc (Vitis vinifera) wine grapes. LWT-Food Science and Technology 2009, 42:1269-1274.

28. Munoz-Espada AC, Wood KV, Bordelon B, Watkins BA: Anthocyanin quantification and radical scavenging capacity of Concord, Norton, and Marechal Foch grapes and wines. J Agric Food Chem 2004, 52:6779-6786.

29. Giribaldi M, Perugini I, Sauvage FX, Schubert A: Analysis of protein changes during grape berry ripening by 2-DE and MALDI-TOF. Proteomics 2007, 7(17):3154-3170.

30. Lijavetzky D, Francisco R, Peng FY, Bravo G, Ibanez A, Oliveros JC, Lund ST, Martinez-Zapater JM: A new GeneChip for grapevine transcriptomic analysis. Aust J Grape Wine Res 2010, 16(Supplemental 1):A51.

31. Weng L, Dai H, Zhan Y, He Y, Stepaniants SB, Bassett DE: Rosetta error model for gene expression analysis. Bioinformatics 2006, 22:1111-1121.

32. Benjamini $Y$, Hochberg $Y$ : Controlling the false discovery rate: a practical and powerful approach to multiple testing. I Roy Stat Soc Ser B (Stat Method) 1995, 57:289-300.

33. Uknes S, Mauch-Mani B, Moyer M, Potter S, Williams S, Dincher S, Chandler D, Slusarenko A, Ward E, Ryals J: Acquired resistance in Arabidopsis. Plant Cell 1992, 4(6):645-656.

34. van Loon LC, Rep M, Pieterse CMJ: Significance of inducible defense-related proteins in infected plants. Annu Rev Phytopathol 2006, 44(1):135-162.

35. Le Henanff G, Heitz T, Mestre P, Mutterer J, Walter B, Chong J: Characterization of Vitis vinifera NPR1 homologs involved in the regulation of PathogenesisRelated gene expression. BMC Plant Biology 2009, 9(1):54.

36. Encinas-Villarejo S, Maldonado AM, Amil-Ruiz F, de los Santos B, Romero F, Pliego-Alfaro F, Munoz-Blanco J, Caballero JL: Evidence for a positive regulatory role of strawberry (Fragaria $\times$ ananassa) FaWRKY1 and Arabidopsis AtWRKY75 proteins in resistance. J Exp Bot 2009, 60(11):3043-3065. 
37. McHale L, Tan X, Koehl P, Michelmore RW: Plant NBS-LRR proteins: adaptable guards. Genome Biol 2006, 7:212.

38. DeYoung BJ, Innes RW: Plant NBS-LRR proteins in pathogen sensing and host defense. Nat Immunol 2006, 7:1243-1249.

39. Hewezi T, Mouzeyar S, Thion L, Rickaue M, Alibert G, Nicolas P, Kallerhoff J: Antisense expression of a NBS-LRR sequence in sunflower (Helianthus annuus L.) and tobacco (Nicotiana tabacum L.): evidence for a dual role in plant development and fungal resistance. Transgenic Res 2006, 15:165-180.

40. Monteiro S, Barakat M, Picarra-Pereira MA, Teixeira AR, Ferreira RB: Osmotin and thaumatin from grape: A putative general defense mechanism against pathogenic fungi. Phytopathology 2003, 93:1505-1512.

41. Busam G, Kassemeyer HH, Matern U: Differential expression of chitinase in Vitis vinifera L. responding to systemic acquired resistance activators or fungal challenge. Plant Physiol 1997, 115:1029-1038.

42. Girault T, François J, Rogniaux H, Pascal S, Delrot $S$, Coutos-Thévenot $P$, Gomès E: Exogenous application of a lipid transfer protein-jasmonic acid complex induces protection of grapevine towards infection by Botrytis cinerea. Plant Physiol Biochem 2008, 46:140-149.

43. Gomès E, Sagot E, Gaillard C, Laquitaine L, Poinssot B, Sanejouand YH, Delrot $S$, Coutos-Thévenot $P$ : Nonspecific lipid-transfer protein genes expression in grape (Vitis sp.) cells in response to fungal elicitor treatments. Mol Plant Microbe Interact 2003, 16:456-464.

44. Laquitaine L, Gomès E, François J, Marchive C, Pascal $S$, Hamdi $S$, Atanassova R, Delrot S, Coutos-Thévenot P: Molecular basis of ergosterolinduced protection of grape against Botrytis cinerea: Induction of type I LTP promoter activity, WRKY, and stilbene synthase gene expression. Mol Plant Microbe Interact 2006, 19:1103-1112.

45. Jeandet P, Douillet-Breuil AC, Bessis R, Debord S, Sbaghi M, Adrian M: Phytoalexins from the Vitaceae: Biosynthesis, phytoalexin gene expression in transgenic plants, antifungal activity, and metabolism. $J$ Agric Food Chem 2002, 50:2731-2741.

46. Dercks W, Creasy LL: The significance of stilbene phytoalexins in the Plasmopara viticola-grapevine interaction. Physiol Mol Plant Pathol 1989, 34:189-202.

47. Pezet R, Pont V: Ultrastructural observations of pterostilbene fungicoxicity in dormant conidia of Botrytis cinerea Pers. Physiol Plant Pathol 1981, 18:213-226.

48. Montero C, Cristescu SM, Jimenez JB, Orea JM, te Lintel Hekkert S, Harren FJM, Gonzalez Urena A: trans-resveratrol and grape disease resistance. A dynamical study by high-resolution laser-based techniques. Plant Physiol 2003, 131(1):129-138

49. Maddox CE, Laur LM, Tian L: Antibacterial activity of phenolic compounds against the phytopathogen Xylella fastidiosa. Curr Microbiol 2010, 60:53-58.

50. Udenigwe CC, Ramprasath VR, Aluko RE, Jones PJ: Potential of resveratrol in anticancer and anti-inflammatory therapy. Nutr Rev 2008, 66:445-454.

51. Fornara V, Onelli E, Sparvoli F, Rossoni M, Aina R, Marino G, Citterio S: Localization of stibene synthase in Vitis vinifera L. during berry development. Protoplasma 2008, 233:83-93.

52. Gatto P, Vrhovsek U, Muth J, Segala C, Romualdi C, Fontana P, Pruefer D Stefanini M, Moser C, Mattivi F, et al: Ripening and genotype control stilbene accumulation in healthy grapes. J Agric Food Chem 2008, 56:11773-11785.

53. Versari A, Parpinello GP, Tornielli GB, Ferrarini R, Giulivo C: Stilbene compounds and stilbene synthase expression during ripening, wilting, and UV treatment in grape cv. Corvina. J Agric Food Chem 2001, 49(11):5531-5536.

54. Conn S, Curtin C, Bezier A, Franco C, Zhang W: Purification, molecular cloning, and characterization of glutathione S-transferases (GSTs) from pigmented Vitis vinifera L. cell suspension cultures as putative anthocyanin transport proteins. J Exp Bot 2008, 59(13):3621-3634.

55. Dalton DA, Boniface C, Turner Z, Lindahl A, Kim HJ, Jelinek L, Govindarajulu M, Finger RE, Taylor CG: Physiological roles of glutathione S-transferases in soybean root nodules. Plant Physiol 2009, 150:521-530.

56. Dixon DP, Skipsey M, Edwards R: Roles for glutathione transferases in plant secondary metabolism. Phytochemistry 2010, 71:338-350.

57. Zenoni SFA, Giacomelli E, Xumerle L, Fasoli M, Malerba G, Bellin D, Pezzotti M, Delledonne M: Characterization of transcriptional complexity during berry development in Vitis vinifera using RNA-Seq. Plant Physiol 2010, 152:1787-1795
58. Kitamura S, Shikazono N, Tanaka A: TRANSPARENT TESTA 19 is involved in the accumulation of both anthocyanins and proanthocyanidins in Arabidopsis. Plant J 2004, 37:104-114

59. Castellarin SD, Pfeiffer A, Sivilotti P, Degan M, Peterlunger E, Gaspero GD: Transcriptional regulation of anthocyanin biosynthesis in ripening fruits of grapevine under seasonal water deficit. Plant, Cell and Environ 2007, 30(11):1381-1399.

60. Ibrahim RK, Bruneau A, Bantignies B: Plant O-methyltransferases: molecular analysis, common signature and classification. Plant Mol Biol 1998, 36:1-10.

61. Schroder $G$, Wehinger $E$, Schroder J: Predicting the substrates of cloned plant O-methyltransferases. Phytochemistry 2002, 59:1-8.

62. Ageorges A, Fernandez L, Vialet S, Merdinoglu D, Terrier N, Romieu C: Four specific isogenes of the anthocyanin metabolic pathway are systematically co-expressed with the red colour of grape berries. Plant Sci 2006, 170:372-383.

63. Costantini E, Landi L, Silvestroni O, Pandolfini T, Spena A, Mezzetti B: Auxin synthesis-encoding transgene enhances grape fecundity. Plant Physiol 2007, 143:1689-1694

64. Hugueney P, Provenzano S, Verries C, Ferrandino A, Meudec E, Batelli G, Merdinoglu D, Cheynier V, Schubert A, Ageorges A: A novel cationdependent $O$-methyltransferase involved in anthocyanin methylation in grapevine. Plant Physiol 2009, 150(4):2057-2070.

65. Deluc L, Quilici D, Decendit A, Grimplet J, Wheatley M, Schlauch K, Merillon JM, Cushman J, Cramer G: Water deficit alters differentially metabolic pathways affecting important flavor and quality traits in grape berries of Cabernet Sauvignon and Chardonnay. BMC Genomics 2009, 10(1):212.

66. Janvary L, Hoffmann T, Pfeiffer J, Hausmann L, Topfer R, Fischer TC, Schwab W: A double mutation in the anthocyanin 5-Oglucosyltransferase gene disrupts enzymatic activity in Vitis vinifera L. J Agric Food Chem 2009, 57(9):3512-3518.

67. Bogs J, Jaffe F, Takos A, Walker A, Robinson S: The grapevine transcription factor VvMYBPA1 regulates proanthocyanidin synthesis during fruit development. Plant Physiol 2007, 143(3):1347-1361.

68. Walker A, Lee E, Bogs J, McDavid D, Thomas M, Robinson S: White grapes arose through the mutation of two similar and adjacent regulatory genes. Plant J 2007, 49:772-785.

69. Coombe B: Adoption of a system for identifying grapevine growth stages. Aust J Grape Wine Res 1995, 1:100-110.

70. Reid K, Olsson N, Schlosser J, Peng F, Lund S: An optimized grapevine RNA isolation procedure and statistical determination of reference genes for real-time RT-PCR during berry development. BMC Plant Biology 2006, 6(1):27.

71. Grimplet J, Cramer GR, Dickerson JA, Mathiason K, Van Hemert J, Fennell AY: VitisNet: "Omics" Integration through grapevine molecular networks. PLOS ONE 2009, 4(12):e8365.

72. Peirson SN, Butler JN, Foster RG: Experimental validation of novel and conventional approaches to quantitative real-time PCR data analysis. Nucl Acids Res 2003, 31:e73.

\section{doi:10.1186/1471-2229-11-7}

Cite this article as: Ali et al:: Berry skin development in Norton grape: Distinct patterns of transcriptional regulation and flavonoid biosynthesis. BMC Plant Biology 2011 11:7.

\section{Submit your next manuscript to BioMed Central and take full advantage of:}

- Convenient online submission

- Thorough peer review

- No space constraints or color figure charges

- Immediate publication on acceptance

- Inclusion in PubMed, CAS, Scopus and Google Scholar

- Research which is freely available for redistribution 